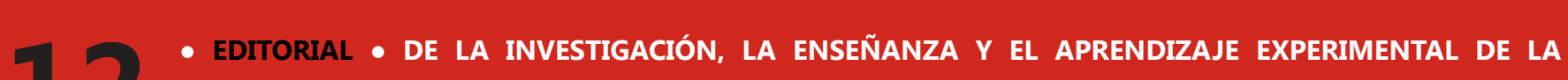
ARQUITECTURA / FROM RESEARCH, TEACHING AND THE EXPERIMENTAL LEARNING OF ARCHITECURE. Amadeo Ramos CarranZa • ENTRE LínEAS • LA ARQUITECTURA COMO MODO DE ENTENDER EL MUNDO. NOTAS DE UN PROFESOR VETERANO / ARCHITECTURE AS A WAY TO UNDERSTAND THE WORLD. NOTES FROM A VETERAN PROFESSOR. Antonio González-Capitel - ARTículos • LA PALABRA DIBUJADA. ANTONIO FERNÁNDEZALBA, PRIMER Y ÚLTIMO MAESTRO / THE SKETCHED WORD. ANTONIO FERNÁNDEZ-ALBA, THE FIRST AND LAST MASTER. Juan Luis Trillo de Leyva • CARVAJAL Y LA VOLUNTAD DE SER ARQUITECTO: LA CONSTRUCCIÓN DEL PROYECTO Y LA BELLEZA EFICAZ / CARVAJAL AND THE WILL TO BE ARCHITECT: THE CONSTRUCTION OF THE PROJECT AND EFFECTIVE BEAUTY. Carlos Labarta Aizpún; Jorge Tárrago Mingo • CIUDAD BLANCA EN BAHÍA DE ALCUDIA. UNA OBRA CON SENTIDO PEDAGóGICO DEL PROFESOR FRANCISCO JAVIER SÁENZ DE OÍZA. 1961-63 / CIUDAD BLANCA IN ALCUDIA BAY. AN EDUCATIONAL WORK BY PROFESSOR FRANCISCO JAVIER SÁENZ DE OíZA. 1961-63. Rosa María Añón Abajas; Salud María Torres Dorado • SEVILLA Y EL SEVILLA 1(1972-2015) / SEVILLE AND THE SEVILLE 1 (1972-2015). Valentín Trillo Martínez • DE LA PROFESIÓN A LA DOCENCIA: LOS VIAJES A INGLATERRA DE MANUEL TRILLO Y LAS VIVIENDAS EN LA MOTILLA / FROM PROFESSION TO TEACHING: MANUEL TRILLO AND HIS TRIPS TO ENGLAND AND THE COLLECTIVE HOUSING IN LA MOTILLA. Amadeo Ramos Carranza; José Altés Bustelo • LA CONDICIÓN TERRITORIAL DE LO URBANO. EN TORNO A LA TRAYECTORIA DOCENTE DE PABLO ARIAS / THE CITY WITHIN THE FRAME OF TERRITORY. ABOUT THE ACADEMIC CAREER OF PABLO ARIAS. Victoriano Sainz Gutiérrez • RESEÑAS BIBLIOGRÁFICAS • MANUEL TRILLO DE LEYVA: LA EXPOSICIÓN IBEROAMERICANA: LA TRANSFORMACIÓN URBANA DE SEVILLA. Alfonso del Pozo y Barajas • MANUEL TRILLO DE LEYVA: CONSTRUYENDO LONDRES; DIBUJANDO EUROPA. Tomás Curbelo Ranero; Manuel Ramos Guerra

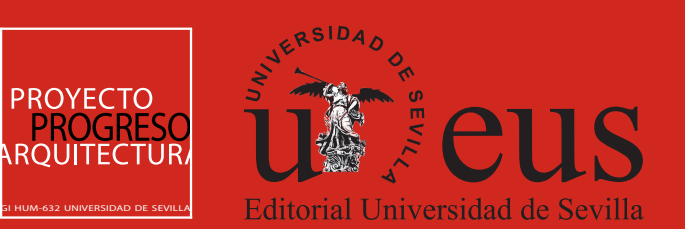

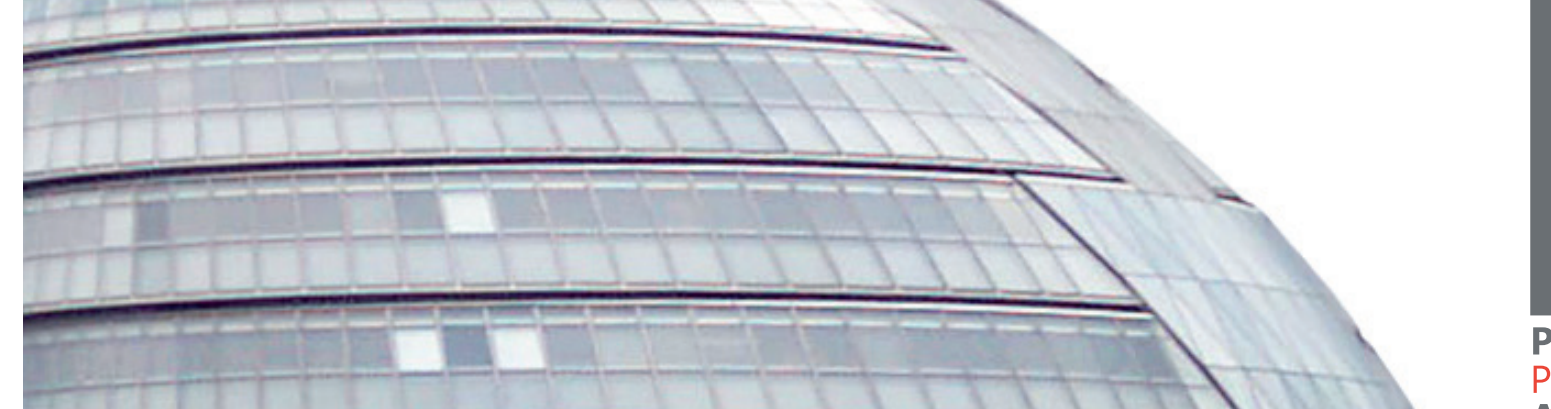

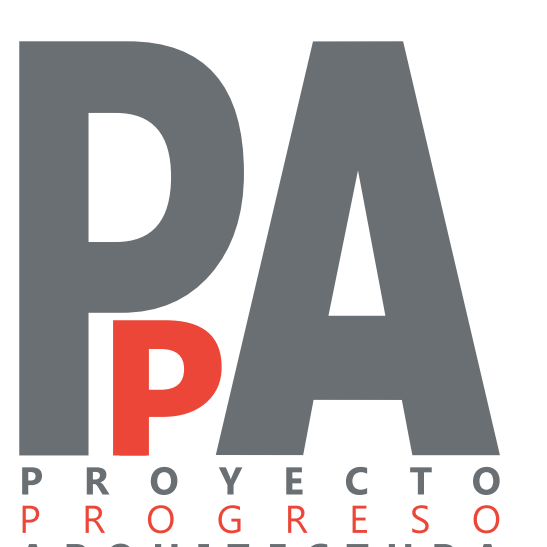

A R Q U I T E C T U R A
ARQUITECTOS Y PROFESORES 
DIRECCIÓN

Escuela Técnica Superior de

Arquitectura. Universidad de Sevilla

SECRETARIA

Dr. Rosa María Añón Abajas. Escuela Técnica Superior de Arquitectura Universidad de Sevilla

Dr. Rosa María Añón Abajas. Escuela Técnica Superior de Arquitectura Universidad de Sevilla. España. Miguel Ángel de la Cova Morillo-Velarde. Escrantectura. Universidad de Sevilla. España

Arquitectura. Universidad de Sevilla. España. Juan José López de la Cruzz. Escuela Tecnica Superior de Arqu
Universidad de Sevilla. España. Germán López Mena. Escuela

Universidad de Sevilla. Españá. Arquitectura. Universidad de Sevilla. Espanan

nica Superior de Arquitectura. Dr. Alfonsad de Sevilla. España .

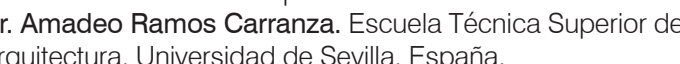

COMITÉ CIENTÍFICO

Dr. Gonzalo Díaz Recaséns. Catedrático Proyectos Arquitectónicos Escuela Técnica Superior de Arquitectura. Universidad de Sevilla. Espan Escusela Mécnica Superior de Arquitectura Universidad Politécnicán de Madrid. España.

Dr. Víctor Pérez Escolano. Catedrático Historia, Teoría y Composición Arquitectónicas. Escuela Técnica Superior de Arquitectura. Universidad r. Jorge Torres $\mathrm{C}$

Técnica Superior de Arquitectura. Universididad de Valenciai España Dr. Armando Dal'Fabbro. Profosessore Associato. Dipartimento di

progettazione architettonica, Facoltà di Architettura, Universitat Instituto

Dr. Mario Coyula Cowley. Profesor de Mérito en la Facultad de
Arcuitectura, del Instituto Superior Politécnico José Antonio Echever Cuba.

Dr. Anne-Marie Chatelêt. Professeur Titulaire. Histoire et Cultures Architecturales. École Nationale Supérieure d'Architecture de Versailles.
Francia CONSEJO ASESOR

Alberto Altes Arlandis. Escola d'Arquitectura del Vallès. Universitat Politécnica de Catalunya. Espana

Dr. José Altés Bustelo. Escuela Técnica Superior de Arquitectura

Dr. José de Coca Leicher. Escuela de Arquitectura y Geodesi

Universidad de Alcala de Henares. España

Dr. Jaume J. Ferrer Fores. Escola Tècnica Superior d'Arquitectura de

Barcelona. Universitat Politècnica de Catalunya. España

Atlántico. Colombia.

Carmen Peña de Urqú́ architect en RSH-P. Londres. Reino Unido.

ISSN-ed. impresa: 2171-6897

ISSN-ed. electrónica: 2173-1616

DOl: http:///dx.doi.org/10.12795/ppa

DEPÓSITO LEGAL: SE-2773-2010
PERIOCIDAD DE LA REVISTA: MAYOY

IMPRIME: TECNOGRAPHIC S.L.
EDITA

AQUETA DE LA PORTADA

Miguel Ángel de la Cova Morillo-Velarde.

DISEÑO DE LA PORTADA

DTOGRAF́́A DE LA PORTADA

, (Londres, junio 2003).

DISEÑO GRÁFICO Y DE LA MAQUETACIÓN

inodríguez

COLABORACIÓN EN EL DISEÑO DE LA PORTADA Y MAQUETACIÓN

Alvaro Borrego Plata.

E.T.S. de Arquitectura. Avda Reina Mercedes, no 241012 -Sevilla.

Amadeo Ramos Carranza, Dpto. Proyectos Arquitectónicos.

EDICIÓN ON-LINE

Portal intormatico https://ojs. publius.us.es/ojs/index.php/ppa/index

Portalinformático G.I.IUM-632http://muw.proyectoprogresoarquitectura.com

hortta://nww.ermático Editorial.us.es/

(c) EDITORIAL UNIIERSIDAD DE SEVILLA.

Calle Porvenir, 27. 41013 SEVILLA. Tfs. $954487447 / 95448745$

๑ TEXTOS: SUS AUTORES.

( $)$ IMAGENES: SUS AUTORES Y/O INSTITUCIONES.

SUSCRIPCIONES, ADQUISICIONES Y CANJE

EVISTa PROYECTO, PROGRESO, ARQUITECTURA

Caltorial Ponivenirsididad de Sevilla. 41013 SEVILLA. Tfs. 954487447 / 95448745

Reservados todos los derechos. Ni la totalidad ni parte de esta revista puede reproducirse otransmititise por ningun procedimiento electrónico o mecánico,
incluyendo fotocopia grabación magnética o cualquier almacenamiento de información y sistema de recuperación, sin permiso escrito del Secretariado de Publicaciones de la Universidad de Sevilla

tores en los artículos firmados son responsabilidad exclusiva de los mismos.

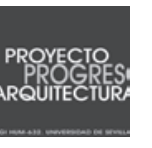

INICIATIVA DEL GRUPO DE INVESTIGACION HUM-632 ittp.//mmw proyectoprogresoarquitecturacon

COLABORA EL DEPAATAMENTO DE PROYECTOS ARQUITECTÓNIICOS hittp://wnw departamento us es/dpetses

\section{revista PROYECTO, PROGRESO, ARQUITECTURA}

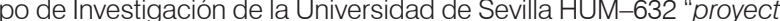
progreso, arqu lecthra t tiene por objetivo compartir y debatir sobre investigación en arquitectura. Es una publicación cientifica con (a) sistema doble ciego-siguiendo los protocolos habituales para publicaciones cientfficas seriadas. Los fitulos, resúmenes y palabras "proyecto, progreso, arquitectura" presenta una estructura clara, sencilla y flexible. Trata todos los temas relacionados con la teoria
la práctica del proyecto arquitectónico. Las distintas "temáticas abiertas" que componen nuestral linea editorial, son las fuentes para de arquitectura. Dur Journal, "proyecto, progreso, arquitectura", founded in 2010, is an inititative of the Research Group HUM-632 of the University of Sevilie and its objective is the sharing and debating of research within architecture. This six-monthly scientific publication, in paper and digital format, publishes original works that have not been previously pubilshed in other journals. Tiene article silection process summaries and key words of articles are also published in English. 作 diverse investigations.

(the accomplishment of

\section{SISTEMA DE ARBITRAJE}

VALUACION EXTERNA POR PARES Y ANÓNIM

En lansejo Eticorial de la revista, una vez comprobado que el artículo cumple con las normas relativas a estilo y contenido indicadas y critica de arquitectura, según el modelo doble ciego.

政 evaluación por correo electrónico, en la dirección que éstos hayan utilizado para enviar el artículo. El director comunicará al autor importantes; no aconsejable para su publicación), así como las observaciones y comentarios de los revisores.
intion Si el manuscrito ha sido aceptado con modificaciones, los autores deberán reenviar una nueva versión del artículo, atendiendo a las demandas y sugerencias de los evaluadores externos. Si lo desean, los autores pueden aportar también una carta al Consejo Editorial en la que indicarán el contenido de las modificaciones del artículo. Los articulos con correcciones import
Consejo Asesor y/o Cientffico para verfificar la validez de las modificaciones efectuadas por el autor.

EXTERNAL ANONYMOUS PEER REVIEW

instructions for authors, the article will be sent to two anonymous experts, within the specific field of architectural investigation and critique, for a double blind review

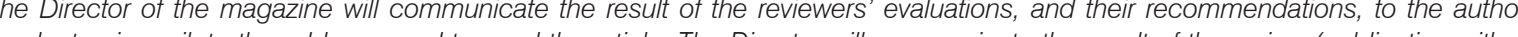
by electronic mall, to the address used to send the article. The Difeclor wil communicate he resull or he review (publicalion withou observations and comments of the reviewers, to the main author. If the manuscript has been accepted with modifications, the authors will have to resubmit a new version of the article, addressing the

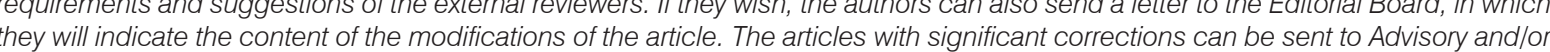
Scientific Board for verification of the valididity of the modifications made by the author

\section{INSTRUCCIONES A AUTORES PARA LA REMISIÓN DE ARTÍCULOS}

NORMAS DE PUBLICACIÓN

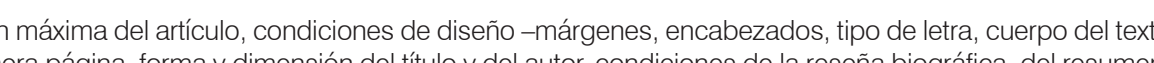
de las palabras claves, de las citas, de las imágenenes -numeración en texto, en pié de imágesenes, calidad de la imagen y autoría procedencia-y de la bibliografía en http://www.proyectoprogresoarquitectura.com

PUBLICATION STANDARDS

instructions to authors: maximum length of the article, design conditions (margins, headings, font, body of the text and quotations, key words, quotations, images (text numeration, image captions, image quality and authorship or origin) and of the bibliography in http " unw.proyectoprogresoarquitectura.com 


\section{SERVICIOS DE INFORMACIÓN}

La Editorial Universidad de Sevilla cumple los criterios establecidos por la Comisión Nacional Evaluadora de la Actividad hivestigadora para que lo publicado por el milsmo sea reconocido como de impacto "Ministerio de Clencia e Innovación. a Editorial Universidad de Sevilla forma parte de la UN E (Unión de Editoriales Universitarias Españolas) ajustáncose a s sistem de control de calidad que garantiza el prestigio e internacionalidad de sus publicaciones. PUBLICATION QUALITY

The Editorial Universidad de Sevilla fulfils the criteria established by the National Commission for the Evaluation of Research Activity (CNEA) so that its publications are recognised as "of impact" (Ministry of Science and I nnovation, Resolution 18939 of 11 Novemb The Editorial Universidad de Serill operates a qualit control system which ensures the prestige and international nature of is publications, and is a member of the U.N. .E. (Unión de Editoriales Universitarias Españolas-Union of Spanish University Publishers.

Los contenidos de la revista PROYECTO, PROGRESO, ARQUITECTURA aparece en:

\section{bases de datos: indexación}

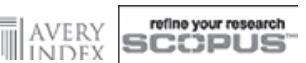 \\ iseceboaros [|[ e-revist@s \\ ProQuest' O Dialnet}

SCOPUS

AVERY. Avery Index to Architectural Periodicals

SSOC (Producida por el CCHS del CSIO)

-REVIST@S (CSIC)

DOAJ, Directory of Open Access Journals
PROQUEST (Arts \& Humanities, full text)

DRIJ. Directory of Research Journals Indexing

SJR (2014): $0.100, H$ index: 0

\section{catalogaciones: criterios de calidad}

RESH (Revistas Españolas de Ciencias Sociales y Humanidades).

Catálogos CNEAl (16 criterios de 19). ANECA (18 criterios de 21). LATINDEX (35 criterios sobre 36)

DICE (CCHS del CSIC, ANECA).

MIAR, Matriu d'I'Informació per a l'Avaluació de Revistes. IDCS 2015: 9,278. Campo ARQUITECTURA (internacional) 24/230

CIRUS, for Scientific Information

ULRICH'S WEB, Global Serials Directory

\section{catálogos on-line bibliotecas notables de arquitectura:}

CLIO. Catálogo on-line. Columbia University. New York

SBD Sistema Bibliotecario e Documentale Instituto Universitario di Architetura di Venezi

OPAC. Servizi Bibliotecari di Ateneo. Biblioteca Centrale. Politecnico di Milano

COPAC. Catálogo colectivo (Reino Unido)
SUDOC Catálogo colectivo (Francia)

ZBD Catálogo colectivo (Alemania)

REBIUN. Catálogo colectivo (España)

OCLC. WorldCat (Mundia)

\section{DECLARACIÓN ÉTICA SOBRE PUBUICACIÓN Y MALAS PRÁCTICAS}

(a) 作 Así nuestra revista garantiza lientificas define el COMITE DE EIICA DE PUBLICACIONES (COPE). publicado, protegiendo y respetando el contenido de los artículos y la integridad de los mismo. El Consejo Editorial se comEn

En cumplimiento de estas buenas prácticas, la revista PPA tiene publicado el sistema de arbitraje que sigue para la selección Consejo Editorial- La revista PPA mantiene actualizado estos criterios, basados exclusivamente en la relevancia cientifica de artículo, originalidad, claridad y pertinencia del trabajo presentado.

Nuestra revista garantiza en todo momento la condifencialidad del proceso de evaluación. el anonimato de los evaluadores y de los autores; el contenido evaluado; el informe razonado emitidos por los evaluadores y cualquier otra comunicación emitida

Igualmente queda afectado de la máxima confidencialidad las posibles aclaraciones, reclamaciones o quejas que un autor su contenido sea fraudulento, serán eliminados o no publicados de la revista PPA. La revista se identifiquen como plagio o mayor celeridad posible. Al aceptar los terminos y acuerdos expresados por nuestra revista, los autores han de garantizar

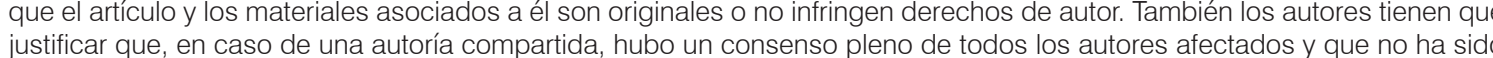
presentado ni publicado con anterioridad en otro medio de difusión.

ETHICS STATEMENT ON PUBLICATION AND BAD PRACTICES

PROYECTO, PROGRESO ARQUITECTURA (PPA) makes a commitment to the academic community by ensuring the ethics and quality of its published articles. As a benchmark, our journal uses the Code of Conduct and Good Practices which, for scientific journals, is defined for editors by the PUBLICATION ETHICS COMMITTEE (COPE). Our journal thereby guarantees an appropriate response to the needs of readers and authors, ensuring the quality of the pu-
blished work, protecting and respecting the content and integrity of the articles. The Editorial Board will publish corrections, clarifications, retractions and apologies when necessand

In compliance with these best practices, PPA has published the arbitration system that is followed for the selection of articles as solely on the scientific importance, the originality clarity and, relevance of the presented article-

Our journal guarantees the confidentiality of the evaluation process at all times: the anonymity of the reviewers and authors; the reviewed content; the reasoned
and scientific boards as required. Equally, the strictest confidentiality applies to possible clarifications, claims or complaints that an author may wish to refer to the

PROYECTO, PROGRESO ARQUITECTURA (PPA) declares its commitment to the respect and integrity of work already published will be eliminated or not published in PPA. The journal will act as quickly as possible in such cases. In accepting the terms and conditions expressed by our journal, authors must guarantee that the article and the materials associated with it are original and do not infringe copyright. The authors will also have to warrant that, in the case of joint authorship, there has been full consensus 
entre lineas LA ARQUITECTURA COMO MODO DE ENTENDER EL MUNDO. NOTAS DE UN PROFESOR
VETERANO / ARCHITECTURE AS A WAY TO UNDERSTAND THE WORLD. NOTES FROM A VETERAN

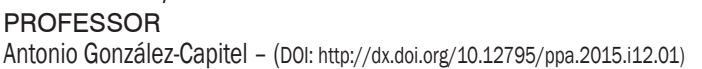

artículos
LA PALABRA DIBUJADA. ANTONIO FERNÁNDEZ-ALBA, PRIMER Y ÚLTIMO MAESTRO / THE SKETCHED WORD. ANTONIO FERNÁNDEZ-ALBA, THE FIRST AND LAST MASTER

CARVAJAL Y LA VOLUNTAD DE SER ARQUITECTO: LA CONSTRUCCIÓN DEL PROYECTO Y LA W AVAAL AND THE WILL TO BE ARCHITECT: THE CONSTRUCTION OF THE PROJECT AND EFFECTIVE BEAUTY
Carlos Labarta Aizpuń; Jorge Tárrago Mingo - (Dol: htpp://dx.doliorg 10.12795/ppa.2015.12.03) CIUDAD BLANCA EN BAHIIA DE ALCUDIA. UNA OBRA CON SENTIDO PEDAGOG ICO DEL
PROFESR FRANCISCO JAVIIER SÁENZ DE OIZZA. 1961 -63 / CIUDAD BLANCA IN ALCUDIA BAY, AN EDUCATIONAL WORK BY PROFESSOR FRANCIZCO JAVIER SAENZ DE OIZZA. 1961-63

SEVILLA Y EL SEVILLA 1(1972-2015)/ SEVILLE AND THE SEVILLE 1(1972-2015)

$$
\text { SEVILLA Y EL SEVILLA 1(1972-2015) / SEVILLE AND }
$$

DE LA PROFESIÓN A LA DOCENCIA: LOS VIAJES A INGLATERRA DE MANUEL TRILLO Y LAS VIVIENDAS EN LA MOTILLA / FROM PROFESSION TO TEACHING: MANUEL TRILLO AND HIS TRIPS TO

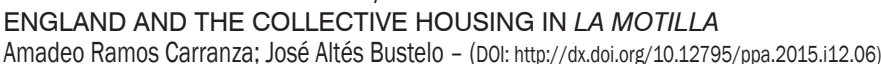

LA CONDICIÓN TERRITORIAL DE LO URBANO. EN TORNO A LA TRAYECTORIA DOCENTE DE PABLO ARIAS / THE CITY WITHIN THE FRAME OF TERRITORY. ABOUT THE ACADEMIC CAREER OF

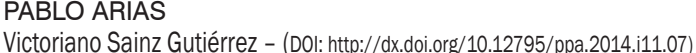

eña bibliográ́ica TEXTOS VIVOS

MANUEL TRILLO DE LEYVA: LA EXPOSICIÓN IBEROAMERICANA: LA TRANSFORMACIÓN

URBANA DE SEVILLA

MANUEL TRILLO DE LEYVA: CONSTRUYENDO LONDRES; DIBUJANDO EUROPA 


\section{CARVAJAL Y LA VOLUNTAD DE SER ARQUITECTO}

\section{LA CONSTRUCCIÓN DEL PROYECTO Y LA BELLEZA EFICAZ}

CARVAJAL AND THE WILL TO BE ARCHITECT: THE CONSTRUCTION OF THE PROJECT

AND EFFECTIVE BEAUTY

Carlos Labarta Aizpún; Jorge Tárrago Mingo
1. Javier Carvajal y Alberto Campo Baeza en una

abril 1982
RESUMEN Javier Carvajal Ferrer (Barcelona 1926-Madrid 2013) aúna magisterio y praxis de tal modo que es imposible acercarse a su obra sin tener en cuenta su docenciaia, al revés, no puede entenderse su discurso en las aulas si, a la vez, no se transita
por sus proyectos. Más aún, para Carvajal el proyecto arquitectónico es el ámbito especifico de la investigación del profesor y el
meccanismo de eficaciaia por el que el profesesional debe dar una respuesta coherente a la teoría y a la praxisi. Este texto pretende mecanismo de eficacia por el que el profesional debe dar una respuesta coherente a la teoría y a la praxis. Este texto pretende
evocar algunas de las virtudes de su obra arquitectónica y de las enseñanzas de su labor docente, ejemplificadas en dos de sus proyectos más emblemáticos. Uno, su proyecto más alabadodo, el Pabellón de España para la Feria Mundial de Nueva York (1964-65)

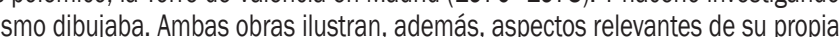
personalidad que inevitablemente se imbrican en su febril actividad como arquitecto y docente, y muestran cómo su firme confianza en la calidad del proyecto sigue siendo la mejor contribucion a la evolucion de la arquitectura. Autenticidad, vocación, voluntad, con lo posible". SUMMARY Javier Carvajal Ferrer (Barcelona, 1926-Madrid, 2013) combined teaching and practice so that it is impossible to
approach his work without considering his teaching and, conversely, we cannot understand his speech in classrooms iff, at the same time, we do not examine his projects. Moreover, for Carvajal the architectural project is the speciific field of the professor's research evoke some of the virtues of his architectural work and the lessons of his teaching, exemplified in two of his most emblematic proects: first, his most praised project, the Spanish Pavilion for the World's Fair in New York (1964-65), which marks fifty years; and the other project-more controversial- - he Valencia Tower in Madrid (1970-73) by researching the rigor and beauty of the plans that he drew himself. Both works also illustrate important aspeects of his personalilty that inevitably overlap in his feverish activity as an lution of architecture. Authenticity vocation, will dedication precision, risor perfection, consistency and illusion are summarized in KEY WORDS Javier Carvajal, teaching, project, plans, New York, Valencia Tower

Persona de contacto/Corresponding autor: clabarta@arquired.es. Departamento de Arquitectura. Área Proyectos Arquitectónicos. Escuela de Inseniería y Arquitectura Universidad de Zarasoza.

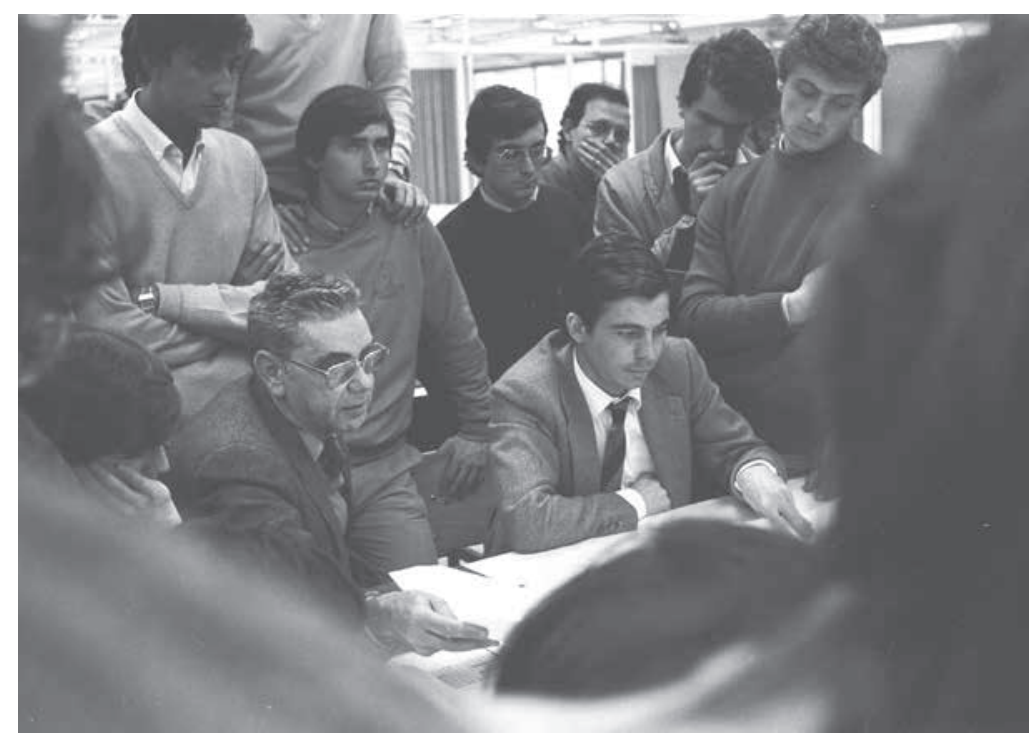

ENSOÑACIÓN Y REALIDAD: UNA REINTERPRETACIÓN DEL LEGADO MODERNO

E ntre los arquitectos y profesores que desde la década de los sesenta contribuyeron a forjar el española parece oportuna la referencia a la a figura de Javier Carvajal Ferrer (Barcelona, 1926-Madrid, 2013). En su caso discurren entrelazadas las trayectorias del arquitecto y del profesor, hasta el extremo de no poder disociar la una de la otra: vocaciones convergentes vividas con generosidad y pasion desbordantes. En sus últimos años como docente solía comentar a los más próximos que su mejor obra, y de la que se sentia mas orgulloso, eran sus alumnos'. No son pocos entre ellos, los que procuran, como académicos de prestigio al cargo de catedras y puestos de responsabilidad docente en las más diversas escuelas de nuestra geogratia, emular la coherencia de su maestro. Asi, sus lecciones -a las que este texto refiere en dos de los proyectos por el más queridos, Pabellon de España en Nueva York (1963-1965) y la Torre de Valencia, Madrid (1970-1973)- se prolongan tanto en alumnos de sus alumnos (figura 1 ).

Docencia y praxis se vinculan en Carvajal a través de la determinante preponderancia otorgada al proyecto arquitectónico a cuya eficaz construcción-desde la emoción y sus obras como en sus alumnos y, de algún modo, en los

la técnica, desde la ensoñación y la realidad- dedicó sus esfuerzos. El proyecto arquitectónico es, para Carvaja el ámbito especifico de la investigación del protesor, a vez que el mecanismo por el que el profesional debe dar una respuesta eficaz, sin advertirse distancia alguna entre teoria y praxis: "la Arquitectura tiene una entidad doble que se expresa en la ideación creativa y en la construcción factica. La Arquitectura no es un arte dibuiado, sino un arte construido".. Esa voluntad de autenticidad se traducla en una autoexigencia extrema en cuantos proyec-

Este texio pretende, a la luz de esos dos proyeclos emblemáticos, evocar simultáneamente, tanto las vifudes de su obra arquitectónica como las enseñanzas volcadas en su labor docente. Para ello recurrimo Jigor y a la belleza de unos planos que el mismo di. de eficacia. Los dos representan la cara y la cruz de Una misma enseñanza. Uno, su proyecto más alabado. Elro, el más polénico. Con suerte y reconocimiento aparontemente dispar, los dos exaclamente igual de ejemplares bajo la perspectiva del proyecto como mecanismo para alcanzar la belleza eficaz. Y los dos tramodo similar -uno desde las in

Carvajal Ferrer Javier. Sobre la génesis del provecto. Pamplona: T6 Ediciones, 1997, pp. 6-7. 2. Ídem. 
arquitecto de dar formaa sus sueños através del proyecto ${ }^{3}$ Ambas obras ilustran como la firme confianza en la calidad del proyecto, sigue siendo la mejor contribución a la "Sueñen con lo posible". Así solía comenzar y terminar Carvajal sus interminables sesiones de correcciones

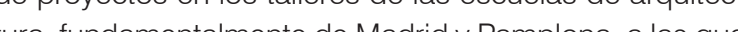
dedicó la mayor parte de su tiempo Su brga y fecun

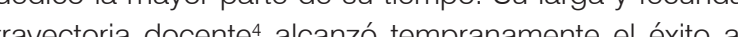

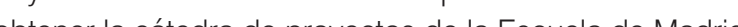
1965, tan solo doce años después de su graduación. Éstas, tan solo doce años despues de su graduación la Escue de Malid lograda por un arquitecto que aperaba en claves de aquitectura modena

Sespués de gú por el contrario la ortodoxia acámica y unto, se-

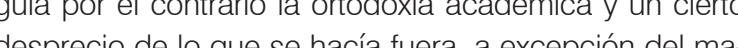
gisterio de algunos profecores 5 Carvajal siempre agradeció las enseñanzas de D. Modesto López Otero 6 . Pese mencionado aislamiento, algunos profesores recuerdan que era el único alumno que, por entonces, manejan

Fullaondo, "quizá el más espectacular y reconocido de a tercera generación madrileña de postguerra" marcada por el "ascendilente racionalista " y "la infiexión orgánicoexprosiva", una genoración que se había ablento camino a base de fuerza contra "I
y culturales"s.

La obtención de su cátedra coincidió en el tiempo con la construcción del pabellón que habria de repre1964-1965. Esta coincidencia dimensiona su intensa y simultánea dedicací́n a la profesión y a la docencia ya propuesta de Carvaja fruto de un concurso, logra, aunque no inicilmonte, concitar el aplauso unánime de la críca naciona e internacional y y encontró en enime abra temprana un reconocimiento más esquiro a los de su generación A la edad de 38 años triunfaba en Nueva York obteriench A la Medalla de Oro frente a competicores como Philip Johnson o Kevin Roche con el pabellón de la Ciudad de Nueva Yok y el pabellón de IBM, respectivamente.

Carvajal sabía que, para un arquitecto, la construcción Cesa idea soña surgía desde el conocimiento profundo de la realidad La innovación técnica y la voluntad de

3. Se utilizza el significado de ensoñación acuñado por Javier Carvajal: "Y cuando digo soñary yablo de ensoñación entiendo el soñar como pensar en aquello que amamos, y sólo atendiendo a lo que nos parece deseable para la felicidad de otros, y para nuestra propia alegría" recogido en "Ensoñación, realidada posibilidad" en Carvajal, Javier: Javier Carvajal. Madrid: Editorial Munilla-Lería, 2000. p. 123.

4. Su dilatada trayectoria docente comenzó un año despoués de obtener el Premio Extraordinario Fin de Carrera en la Escuela de Arquitectura de Madrid. En 1954 es nombrado profésor raxiliar y yoco después, entres 1955 y 1957 estará pensionado en la Academia de Bellas A Artes de Roma. En 1960 será profeseso carso de una cátedra de proyectos sustituyendo a Luis Vilanuuva, contando con las colaboraciones de Garcia de Paredes, Antonio Fernández Allba y Antonio

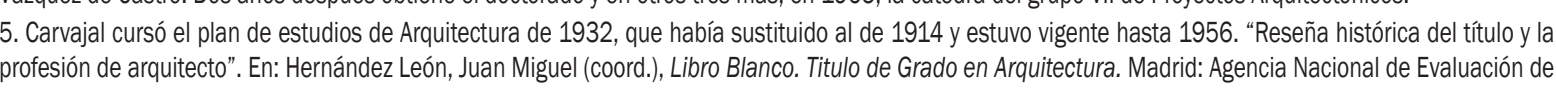
a Calidad y Acreditación, 2005 .

6. Carvajal, Javier. "En memoria del arquitecto D. Modesto López Otero". En AA.W.:. . Carvajal Arquitecto. Madrid: Servicio de Publicaciones del COAM, 1991. p. 43.

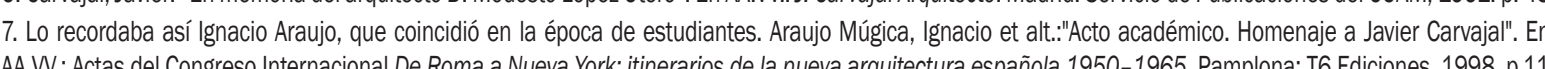
A.W.W: Actas del Congresoso Internacional De Roma a Nueva York: itinerarios de la nueva arquitectura española 1950-1965. Pamplona: T6 Ediciciones. 1998, p.11

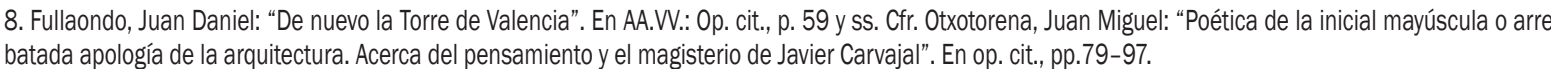

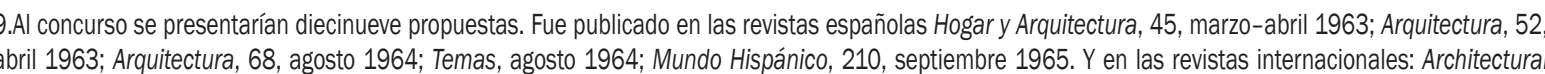
Forum, 120, June 1964; Architectural Record, 136, July 1964; Engineering News Record, 173, July 1964; Progressiviv Architecture, 46, December 1964; Werk 51, December 1964; Arts Magazine, 39, March 1965; The Connoisseur, 160, October 1965; Zodiac, 15, May 1966, entre otras. De igual modo, la prensa

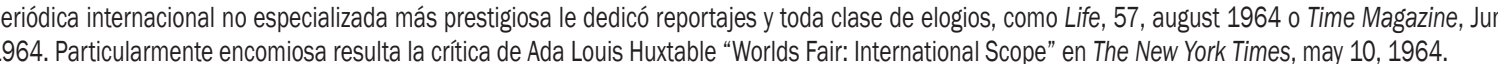

de su tiempo fueron siempre objeto de su mayor interés. Con el pabellón de España en la Feria Mundial de Nueva York'10 inicia su investigación sobre las posibilidades prelabricados de fachada. Investigación que, en la de-

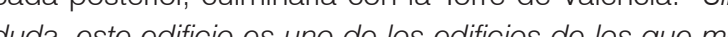
ciento más satisfecho, de cuantos he construido La Tore está bien, a mi entender fuerte cha con buenas pintas

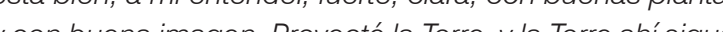
ycon bu a

as, así como su magirter bebía mento, nos segura des mbrando a obra de lose momaestros: de Gropirs, de Le Corbier de Mies, de Aalto de Neutra de Sarinen. de Terrani de Brevery de tanto otros, en los que inturímos y vérmos caminos pana seguir ideas por las que luchar principios que defender"12. Al conocimiento de estos maestros contribuirí decisiva mente su estancia en la Academia de España en Roma entre 1955 y 1957 Y junto a ellos, Carvajal referírí también a maestros más cercanos cuyas enseñanzas se encuentran igualmente en estas primeras obras: "Nuestros jóvenes maestros de entonces, casi de nuestra misma edad, se llamaban Coderch Sota Sáez de Oíza Fisac Cabrero, Aburto y Blanco Soler. Sert Aizpurua, Candela y toda la serie, ya remota de los jóvenes de ante guerra plásticas y constructivas de los elementos de hormigón tor a los estudibe: "Pero a noso-

de quienes queríamos recoger el testigo y a los que quismos unir nuestra singladura de arquitectos: con Molezún en cabeza, con Julio Cano, Corrales, García de Paredes, y

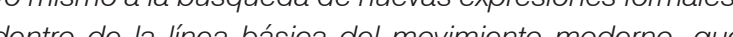

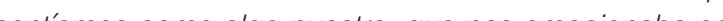
mayor fuerza y compromiso que las antigua retéricas de hs estilos o de los ejemplos históricos, que pod'́n mover y mueven a la admiración pero no a quepetición porer yu realidad arcunstancia no nos mostaba ning, porque tud con nuestra realidad"1.

Estos dos proyectos marcan, respectivamente, el inicio y final de la segunda etapa de su trayectoria'14, acaso la más genuina ${ }^{15}$. El arquitecto, que había desestimado ofertas para haber emprendido la aventura americana tras su éxito en Nueva York, confirma con el proyecto de la torre su fe y confianza en la bondad de la arquitectura como antídoto ante toda suerte de contingencias, sujetas a modas o criticas ocasionales La Torre de Valencia su pone para él prueba más exigentey con ella su lección menos comprendida La voluntad por alcanzar una belleza eficaz, alejada de tópicos coyunturales, simultáneamente enraizada en la tradición y abierta a una modernidad que había abrazado ya en la década precedentite le sostiene con el concurso de un esfuerzo titánico (figuras 2 y 3 ).

La Torre de Valencia, todavía hoy, ejemplifica, como ninguna otra obra de Carvajal, las palabras con las que concluía su última lección académica en la Escuela de (1991:

10. Dos textos recientes abordan el Pabellón de España en Nueva York: Bernal López-Sanvicente, Amparo: "Un espacio para la vanguardia. Nueva York 1964" En Pozo Municio, José Manuel (ed.): Las exposiciones de arquitectura y la arquitectura de las exposiciones. La arquitectura españolal y las exposiciones inter nacionales (1929-1975). Pamplona: T6 Ediciones, 2014. pp. 167-174. Y Oxxotorena Elicegi, Juan Miguul: "Utopía o nostallia, tradición o traición. Sobre Javier Carvajaly y el Pabellón español de la Feria Mundial de 1964 en Nueva York". En Pozo Municio, José Manuel (ed.): 1964/65 New York World's Fair, The Spanis 11 Carvaial Ferrer Javier "Torre de Valencia”" En

En Javier Carvajal. Op. citt, p. 110

12. Carvajal Ferrer, Javier: Curso abierto. Lecciones de arquitectura para arquitectos y no arquitectos. Madrid: Servicio de Publicaciones. Colegio Oficial de

Carvajal" en Javier Carvajal. op.citt, pp. 82-85.

15. A esta etapa pertenecen tambiein la Casa Carvajal (1964-66) y García-Valdecasas (1964-66), ambas en Somosaguas, Madrid. 16. Los proyectos del Panteón de los españoles en el Cementerio del Campo de Verano de Roma y el Pabellón de España en la XI Trienal de Milán, ambos en 1957 y con J.M. Garclia de Paredes, inician y confirman una trayectorial moderna por l q que discu 

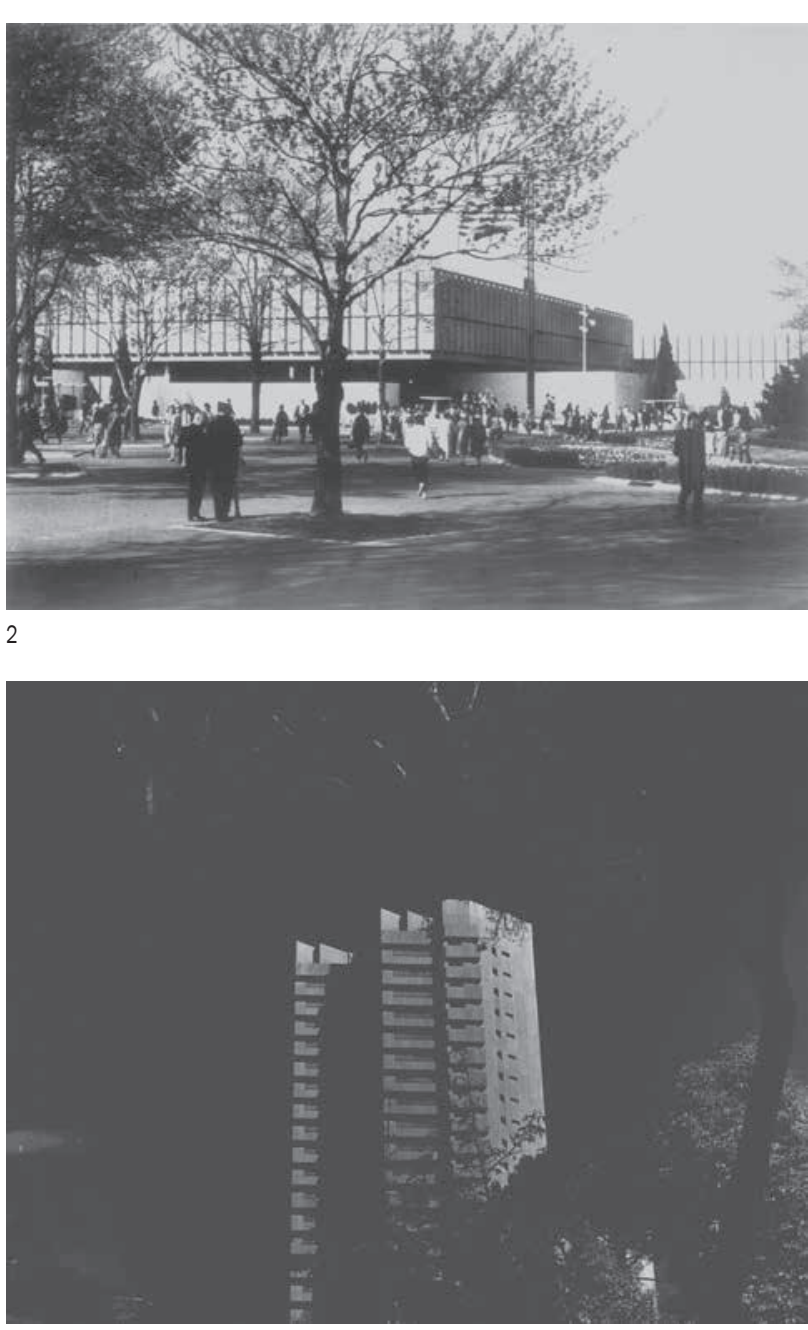

Nunca quedan terminadas las tareas que los hombres emprenden". Una voluntad de permanencia, de superación de las claves del tiempo, que comienza desde las únicas herramientas de que dispone, el dibujo como lenguaje y expresión del arquitecto.

EL PROYECTO COMO MECANISMO DE EFICACIA: DIBUJOS COMENTADOS Y LA ABSTRACCION DE LO CONCRETO

El proyecto para Carvajal nunca es un fin en sí mismo que pueda tender, como tantas veces ocurre, a la autocomplacencia. El proyecto no sólo es el medio para alcanzar la eficacia y la belleza sino que se constituye en mecanismo de éstas: "Porque el proyecto no es otra cosa que un mecanismo de eficacia para alcanzar la obra construida,

2. Pabellón de España. Feria Mundial de Nueva Yoor

de Valencia, Madrid. 1970-73.

4. Planta de ingreso/Main entrance plan. $80 \times 110 \mathrm{~cm}$ Escala 1:100. 5. Detalle. Planta de ingreso/Main entrance plan.
$80 \times 110 \mathrm{~cm}$. Copia sobre papel vegetal. Plano $63-$ 14-28. A-14. Escola $1: 100$

auténtico fin en el cual la arquitectura se concreta"17. Del mismo modo, el dibujo se entiende como la herramienta al servicio de esta finalidad.

Un repaso por los dibujos originales del pabellón nos alerta de la importancia que Carvajal otorga a la representación gráfica de sus proyectos y trasladaba a Campo Baeza de "poner cotas al air"18 en su patente Campín de que el dibujo albergue toda la nu pación potencialidad de hecho construido. El dibujo de las planpos

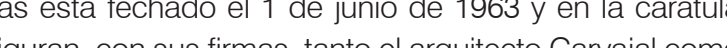
dilu con econocimiento a sula collo fin de consegur la

El trazado de las plantas, el mobiliario, las líneas de cotas así como toda la información de los elementos expositivos conforman un cuadro que constituye, en sl mismo, un documento valioso. Pero este no es el fin de arquitecto como él mismo reconoce y solía insistir a sus alumnos: "Las líneas que conforman los planos no tienen ningún valor artístico en símismas, ni es su fin tenerlo, sino el de ser expresión abstracta de la realidad futura que re presentan"19.

La riqueza que aporta a la construcción de la planta la disposición del mobiliario así como las diferentes definiciones sobre materiales o elementos expositivos, no enturbia, sino que fortalece, la abstracción de una composición heredera de los postulados de la modernidad que Carvajal abraza en esta obra sin necesidad de renuncia a las más hondas raíces de la tradición arquitectónica española, culta o popular. De este modo, las líneas rectas, los cuadrados, los rectángulos y los círculos, todas ellas figuras geométricas básicas, se disponen con distintos tamaños de acuerdo a las necesidades a las que siven "desordenadamente". Según los criterios compositivos, reformulan unas nuevas relaciones de orden que ya no

\section{Carvajal Ferrer, Javier. 0..cit, p. 105.}

18. Campo Baeza, Aliberto. Op.citt, p. 82.

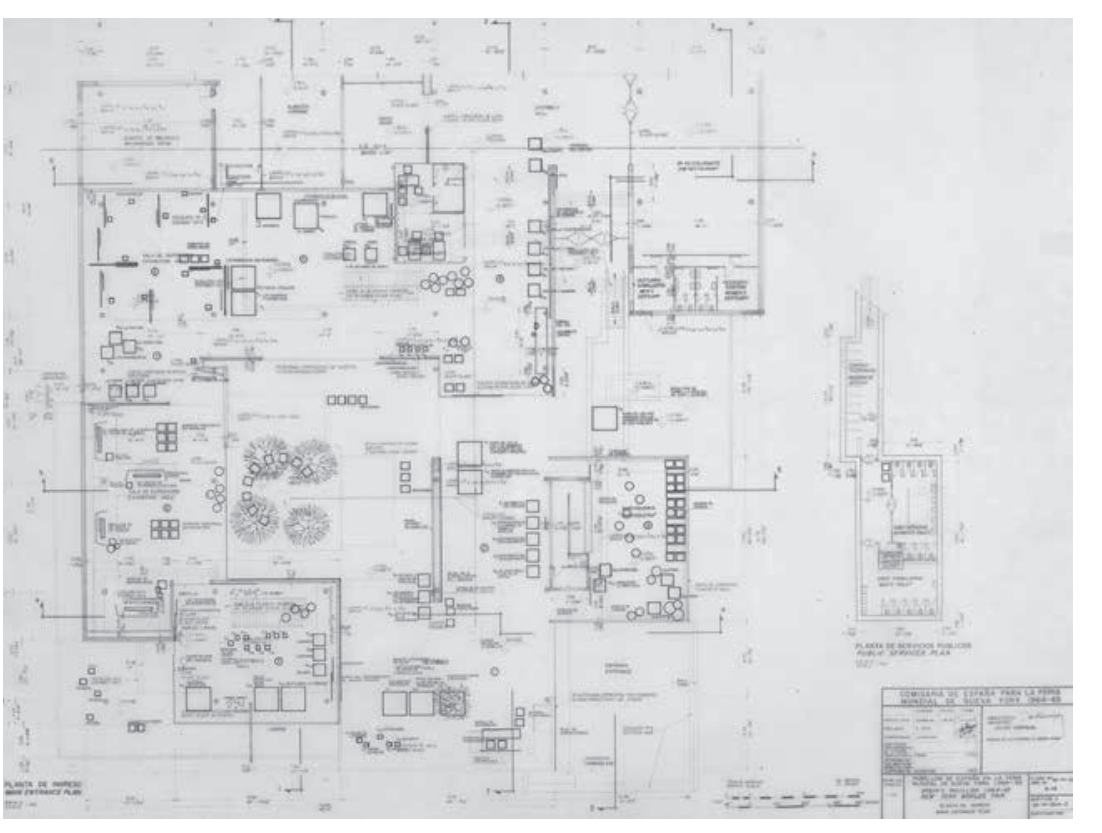

tienen que ver con la jerarquía sino con la clasificación que no refieren a la igualdad sino a la equivalencia $\mathrm{Cua}$ quiera de los fragmentos de las plantas puede leerse bajo esta perspectiva. El magisterio de Canvajal resuena en la composición de cada uno de ellos y, desde ellos, el coniunto on la manifiesta voluntad de que aś contribuse a ordenar todo el mundo a su alrededor.

Estimula observar cada uno de los rincones de la plantas que tienen la capacidad de evocar y anticipar el recorrido del visitante, comprobando, ya desde el estadio del proyecto, las informaciones entrelazadas que Carvaal acota y define con minuciosidad, como por ejemplo: "asientos de terciopelo sin respaldos", "un monie de Zurbarán (la iglesia)", "tarima", "embocadura de madera", "recipientes de barro", "sillón frailero", "la Condesa de Chinchón de Goya (la nobleza)". Todo ello entremezclado y dispuesto en el plano con las cotas de las plantas, e dibujo de los cerramientos y pilares, las precisiones técnicas y de materiales o los ejes de replanteo. Asi se recoge,

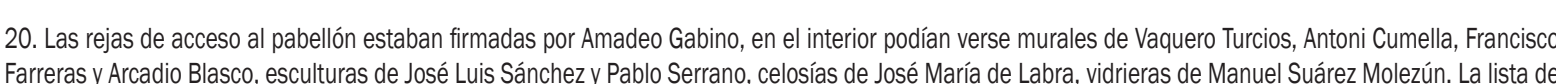

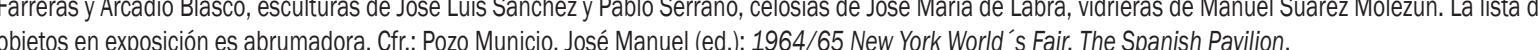

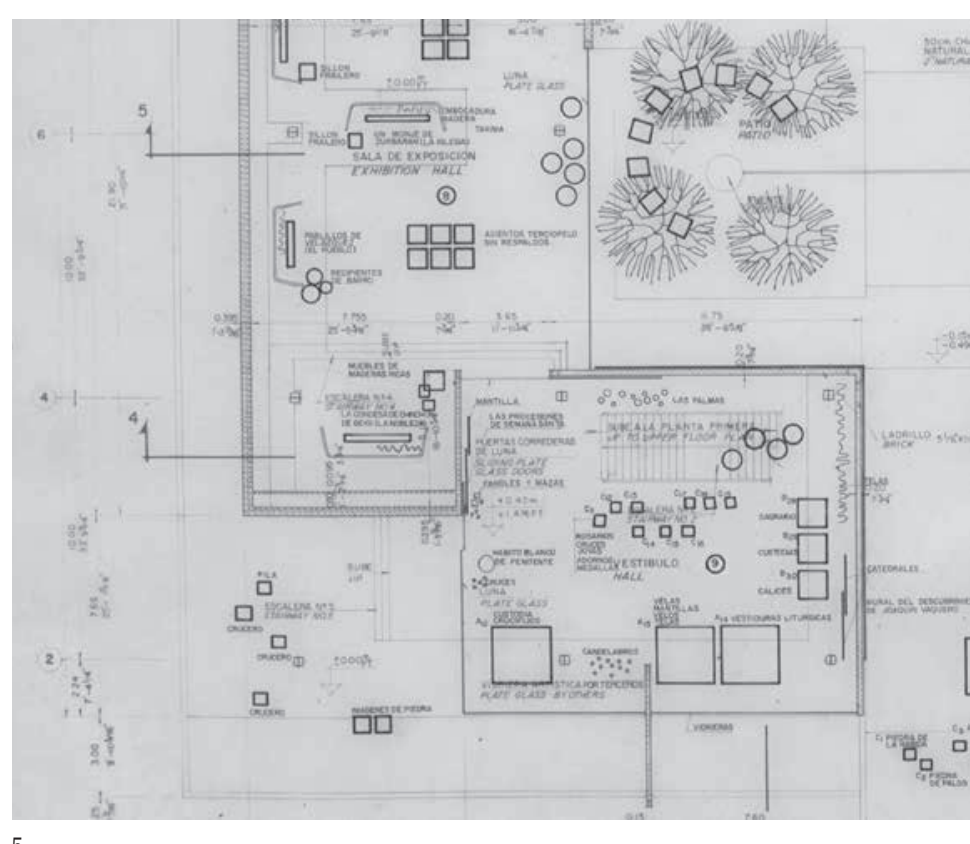

entre otras informaciones, en el plano de la planta de ingreso para la Sala de Exposiciones/Exhibition Hall número 8 (figura 5). Y así, pormenorizadamente, se repite esta esgia en todos y cada uno de los espacios proyectados De este modo, el arquitecto manejaba con destreza los elementos que convertían el espacio en un lugar fantástico y exótico: el visitante se enfrentaba a una atmósfera compleja pero sosegada, de luz intensa y culde un patio y de visiones diagonales y perspectivas amplias. La cantidad de objetos y piezas de arte se desplegaban unas veces integrándose en la arquitectura del pabellón -rejas, murales, vidrieras, esculturas o celosias encargadas a artistas para la ocasión- y otras en mesas, vitrinas o por el suelo ${ }^{20}$. Un espacio donde era dificil distinguir qué era y que no era exposición Carvajal habla empleado ya mecanismos de proyecto similares, pues por entonces habia diseñado varias tiendas 
6. Elementos prefabricados de hormigón armado para fachadas. Pre-cast reinforced concrete elevegetal. Plano 63-74-23. A-11. Varias escalas. 20 de abril de 1963. 7. Secciones de muro. Wall sections 8-8, 9-9. 80x100. Copia sobre papel vegetal. Plano 63-74

3. Sección. Tinta sobre papel vegetal. Firma de lápiz.
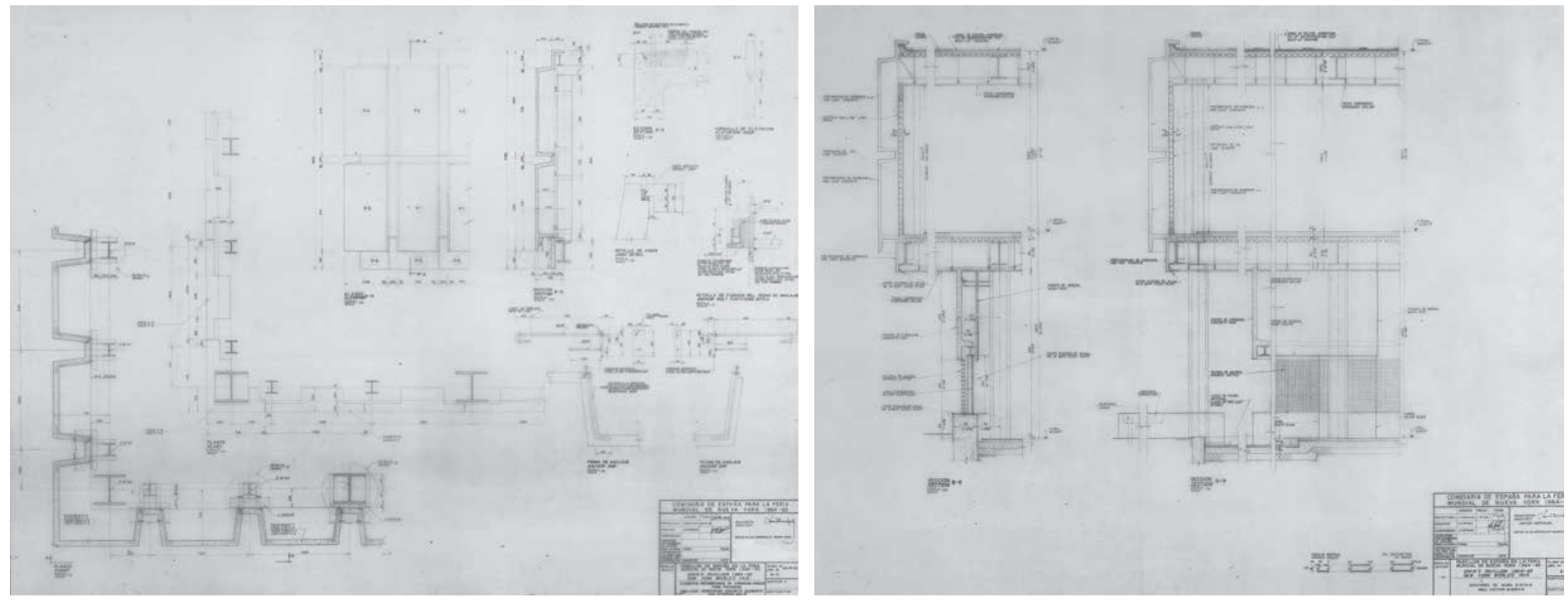

De este modo están también construidos los dibujos de las plantas del pabellón. Trozo a trozo, articulación a artiarquitecto desde el origen: "Ya sé cómo el trabajo va a evolucionar, incluso en sus detalles menores, incluyendo su amueblamiento, pero me es difícil traducir mis intenciones y emociones" 23 .

Carvajal enseña que aquel que sabe dibujar bien, es decir, con enficacia gráficay precisión técnica, podrá conscho construido como fin último yúnico de la aquitectura.

-

DETERMINACIÓN CONSTRUCTVA DIBUJO ARQUITECTÓNICO: EL ORDEN VISUAL DE LOS MATERIALES

De la ingente colección de planos del proyecto, merece la pena detenerse en unos pocos en los que se ejemplifca su ideación, su desarrollo y su construcción ${ }^{24}$. Bajo la aparente casulidad y vibracín de la planta se esconde como mecanismo de orden, el módulo. Los pilares me tálicos se disponen en una malla de diez por diez metros

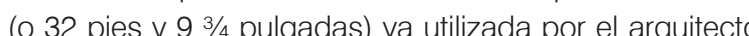
en su propuesta para otro Pabellón de España meno conocido el del concurso para la Bienal de Venecia de 195625. En ambos casos, esta decisión lleva implícita una razón funcional. conseguir la diafanidad que precisan las salas de exposiciones además de hacerlo con un orden interno que afecta a toda la obra de principio a fin.

Los perfiles utilizados son en su mayoría WF (Mide Flange) para cualquier elemento vertical y S (Standard) para cualquiera horizontal según la norma americana, más parecidos al HEB y al IPE europeos. Los foriados se resolvían mediante una chapa colaborante para lograr de ese modo plazos de ejecución relativamente cortos. En el plano 63-74-23. A-11 "Elementos prefabricados de hormigón armado para fachadas" se describe cómo el armazón metálico se revestía después en toda la planta superior con unas piezas de $7 \mathrm{~cm}$ de espesor anclada

sobre una subestructura, también metálica, modulada cada $125 \mathrm{~cm}$, con un anclaje diseñado para permitir e profundich pron de 5482 m 1 a ale alura, proporcionaba al volusas, una composićn regur y seve que ju de maa sombra y vibracín obtenida mediante lu exhaustiva de la misma pieza Éstas se proyectaban a exterior de los forjacos sutimente, exactamente $40 \mathrm{~cm}$ para acentuar una linea continu de sombra, acaso para aligerar e invertir su percepción grave (figura 6). Canvaja subordina les decisiones constructivas a los intereses $v$ subor

En efecto, el pabellón podía parecer discreto, hermético, de apariencia contradictoria, de muros encalados como los de la arquitectura vernácula en su planta baja. y de una cultivada y precisa seriación en las piezas re petidas de los volúmenes que se disponían sobre ellos. Las decenas de planos de secciones constructivas que se dibujaron muestran exhaustivamente cada ligera vaunas lamas correderas- sobre la generalidad de los muros enfoscados de cal que recorren y cierran la planta de ingreso (figura 7).

El plano del suelo no era un plano horizontal sino que dibuiaba una topografía fiel tanto a las características físcas del lugar como a las distintas tensiones que el arquitecto creaba de acuerdo a esas demandas programátcas y espaciales (figura 8).

Esta realidad se evidencia hasta el extremo de que su sobria contundencia exterior no permitía adivinar la riqueza de secuencias que definen las secciones. Los espacios se comprimen y expanden, en ocasiones no más de dos o tres escalones, en continuidad con las visuales diagonales abiertas hacia el patio central y los patios que jalonan el recorrido, no exentas de juegos y significados, ayudando inconscientemente al visitante a percibir su paso a los distintos espacios del proyecto y disolviendo

23. Carvajal Ferrer, Javier: Sobre la genessis del proyecto. op.cit., p. 16.

24. El Archivo General de la Universidadd de Navarra custodia el Fondo Javier Carvajal Ferrer, en el que se incluye el Proyecto 443 , con una colección incompleta de mäs de 180 planos del proyectio del Pabellon de Nueva York, entre orignales y copias, además de fotografías y diapositivas. 25. En este caso el orden
metros entre soportes. 


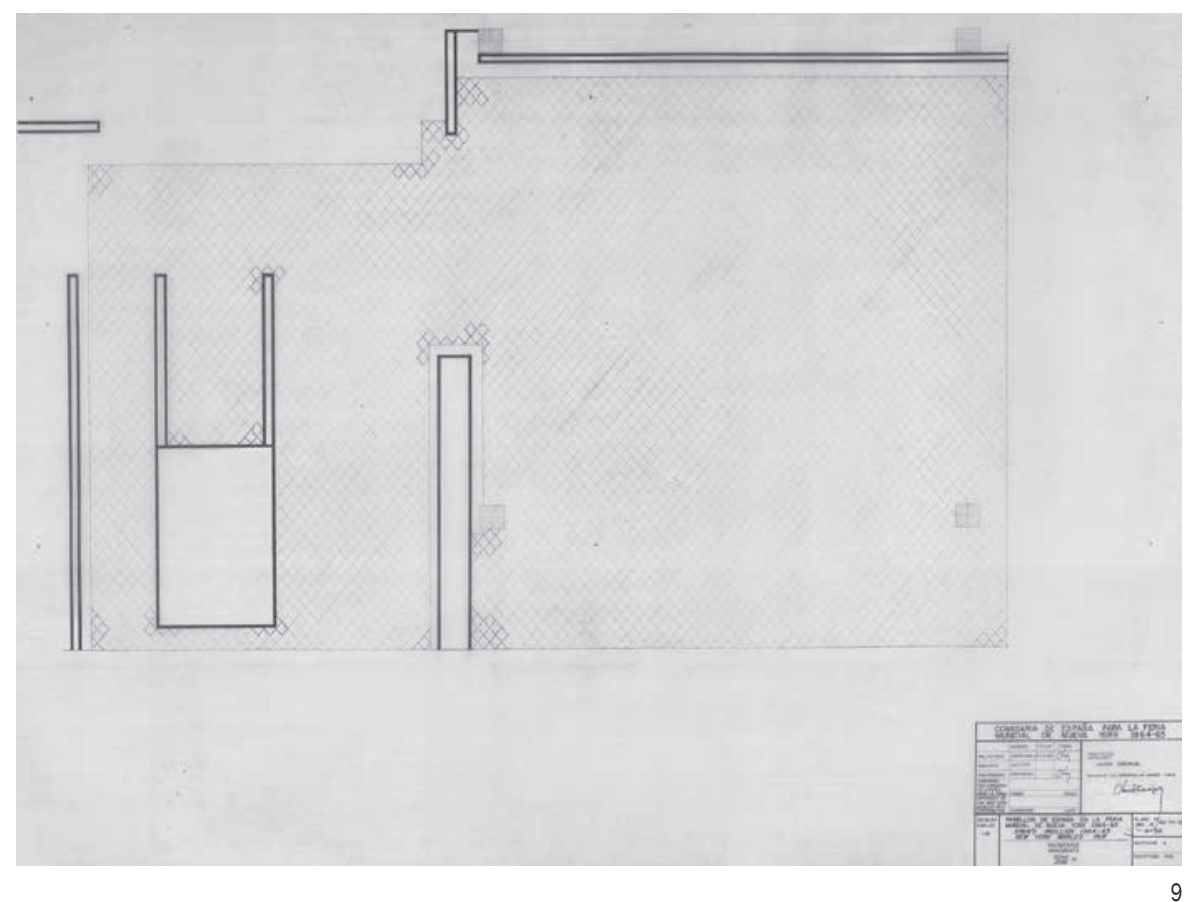

9. Pavimentos. Pavements. Zona / Zone 14. 63-7410. Maqueta de trabajo de la Torre de Valencia. 11. Fotografía de obra con correcciones en rotula res de color en la coronación de la Torre Valencia. 12. Torre de Valencia. continente y contenido. Emulando, de alguna manera, a la arquitectura nazarí de La Alhambra de Granada que tanto admiraba, a la que se refería con frecuencia, y que Tanto los techos de reminiscencias mudéjares, formados por un artesonado a base de piezas cuadradas de madera oscura de nogal, como la iluminación artificial estaban cuidadosamente diseñados en varios planos, con toda clase de detalles, para crear efectos dramáticos ${ }^{26}$. Los pavimentos se trataron como suelos tradicionales de cerámica oscura, de formas y despieces estudiados en todos sus encuentros, pieza a pieza, sin excepción ${ }^{27}$. Nada se deja al azar; todo se dibuja (figura 9)

Carvajal reconocía haber contado con "la tradición como punto de partida, como fidelidad al tiempo presente" $y$ haber sido a través de la arquitectura un "resonador" de la situación de un país, quizá no a la altura del desarrollo técnico que si pudo encontrar en Nueva York, pero con una intensa cultura "en marcha y hacia el futuro"ze. Las investigaciones plásticas y técnicas con las piezas de hormigon prefabricado que habia ensayado, todavia arquitecto, que salía de la España autárquica, participaba, al poco tiempo de llegar a Nueva York, en una inolvidable tertulia en el club de Harvard de la Quinta Avenida con el maestro alemán quien le dijo: "Le puedo dar un consejo. Si quiere tener éxito con su Pabellón no use ni podía esper ni la prefabricación". Y al decirle Carvajal que no de él, Mies le contestó: "Al contrario, soy el único que se lo puede dar. Yo fui el inventor de esas cosas y mire usted (dijo señalando con un amplio gesto de su mano el panorama de la Quinta Avenida), lo que han hecho con mi invento"29. Por fortuna Carvajal se mantuvo fiel a sus convicciones y el pabellón y él mismo obtuvieron, por fin, el reconocimiento unánime.

SOLVENCIA TÉCNICA VERSUS CONTINGENCIA COYUNTURAL: EL PROYECTO COMO ALIADO El profesor Carvajal insistía repetidamente en sus clases la necesidad de que los proyectos estuviesen perfectamente definidos constructivamente alejando, de este modo cualquier sospecha contingente o arbitraria: "arquitectura es tan solo lo que se construye, o lo que tienhabrian de superar la reticencia de Mies van der Rohe. El en sus documentos la virtualidad arquitectónica"so. La

26. Carvajal conto con la colaboración de la norteamericana Display Studios Inc. para la instalación interió

27. Se conservan 3 rollos de planos con planos marcadores de despiecessy de desarrollos de pavimentos segun zonas. Fondo Javier Carvajal. Archivo General de la Universidad de Navarra. Caja $443(6)$

28. Diario ABC: Homenaje al arquitecto Javier Carvajal, Madrid: 19 de diciembre 1964, p. 97.

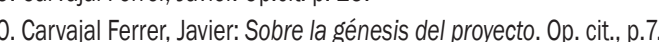
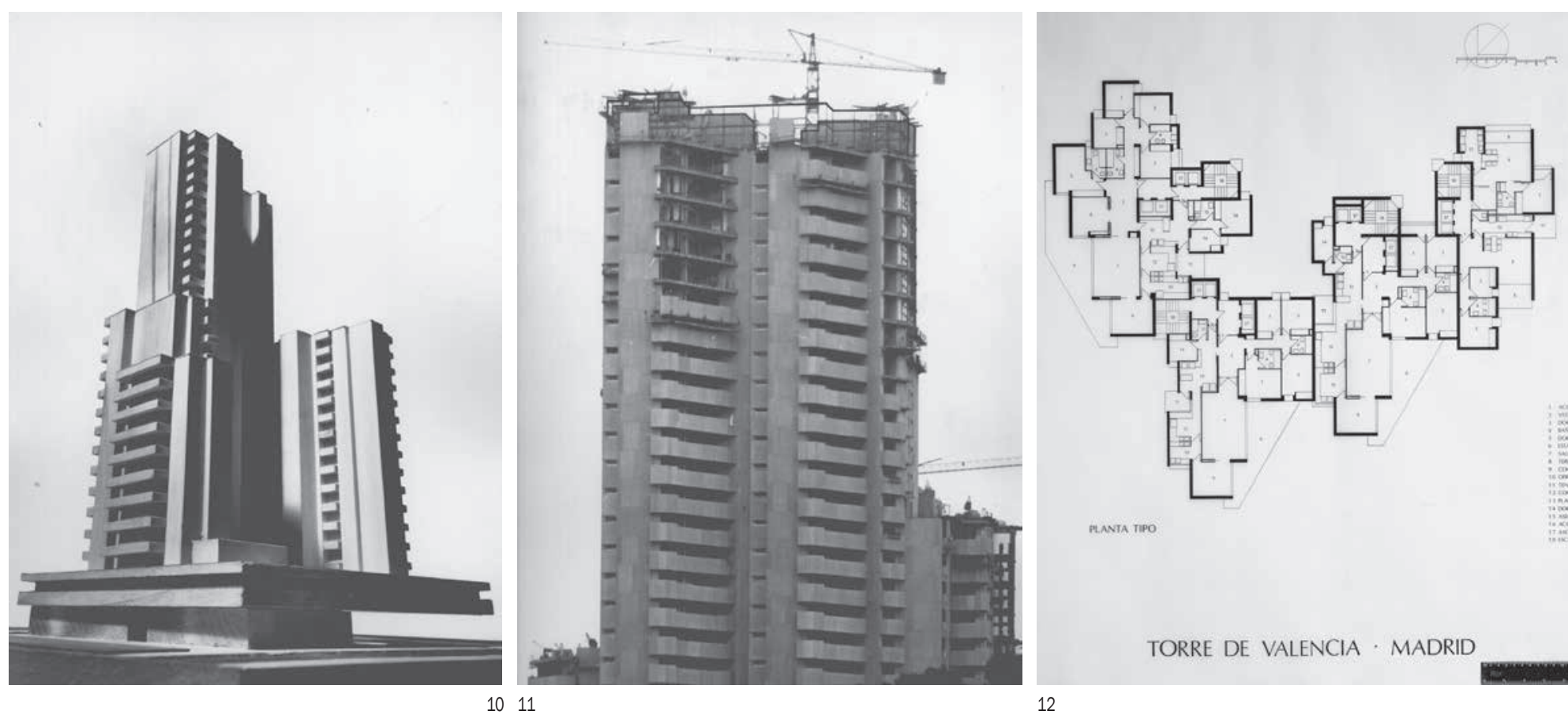

solvencia técnica adquirida con las obras del pabellón implementaría en Carvajal una cualificación técnica superior a la de sus compañeros de generación, a menu-

al estado previo del solar. La voluntad de esculpir una torre en el aire le lleva a verificar el resultado plástico desde el inicio del proyecto. Las imágenes de la maqueta desvelan la verosimilitud de una arquitectura definida en su obra ${ }^{31}$. Esta seguidad alcanzaca en Nueva York contin plantas (veintio ho sobre vasante), deteminaron ta solución estructurat verticl con un solucín mixta de pitares met́licos y núcleos de aniostramiento contra vientode homigón armado, materia tambén uilizado para resolver la estuctua horizontat con fojodos nevados bidireccionates. La voluntad de que todo el proyecto fuera resuelto con un único material, el hormigŕn, cualquira de las modalidaces derivactas de su proceso constructivo, llevó al arquitecto a continuar la investiga ción con los paneles prefabricados ya experimentada con éxito en el pabellón. En este caso, todos los recubrmientos pétreos de las fachadas se realizaron con paneles prefabricados de piedra de Colmenar, anclados a la estructura horizontal

Junto a los planos de proyecto utilizó las maquetas Jomo exí́ a us alumnos- e, incluso las pruebas sobre fotografías de obra como mecanismos de verificación de la solución, difícilmente imaginable por lavibacin de sus sombras (tgura 10). Sin ducla la contraba envulto levaŕan a arquitecto a encontrar, cabe con más precisión la solución más satisfactoria La esbelta figura de la tore en constuccín energe de zócalo compuesto por las dos plantas de oficinas, solventando aś la tensín del encuento ente los planos verticaly horizontal mediante una planta de ingreso fluida que libera 50 por 100 del total del solar dejando amplios espacios sin ocupar (figura 11).

sistemas prefabricados relaciona ambos proyectos. Tanto en la plataforma horzontal del pabellón como en la construcción vertical de torre, Canvajal utiliza estrategias que había ensayado otros proyectos ${ }^{32}$ y que a su vez, referín a esos 'maes tros cercanos' como Coderch. Estas investigaciones espaciales se reinterpretarían unos años después en las plantas de la Torre, que dibujan patios on el aire y proponen unas viviendas unifamiliares en altura (figura 12). Cada vivienda dispone de acceso independiente y vistas

31. Asi refierer Julio Cano Lasso: "Por su calidad de ejecucioion es muy superior a medio tecnologico en que se produce y a los profesesionales que Iuchamos todos los dias por lograr una ejecución aceptable de nuestras obras nos sorprende el grado de perfección alcanzado en la obra de Carvajal". "Sobre la arquitectura de Carvajal" en Javier Carvajal. 0p.cit. p. 12. 32. Junto a las investigaciones espaciales de los patios del pabellón cabe recordar las articulaciones trazadas entre los muros de las casas Carvajal y García
Valdecasas en Somosaguas, 1964-66. 
13. Vista de la Torre de Valencia en construcción

14. Fotografía de la Torre de Valencia tomada desde la C Cl Alcala que
aparecida en ABC.

15. Fotografía de la Torre de Valencia tomada desde el Parque del Retira.

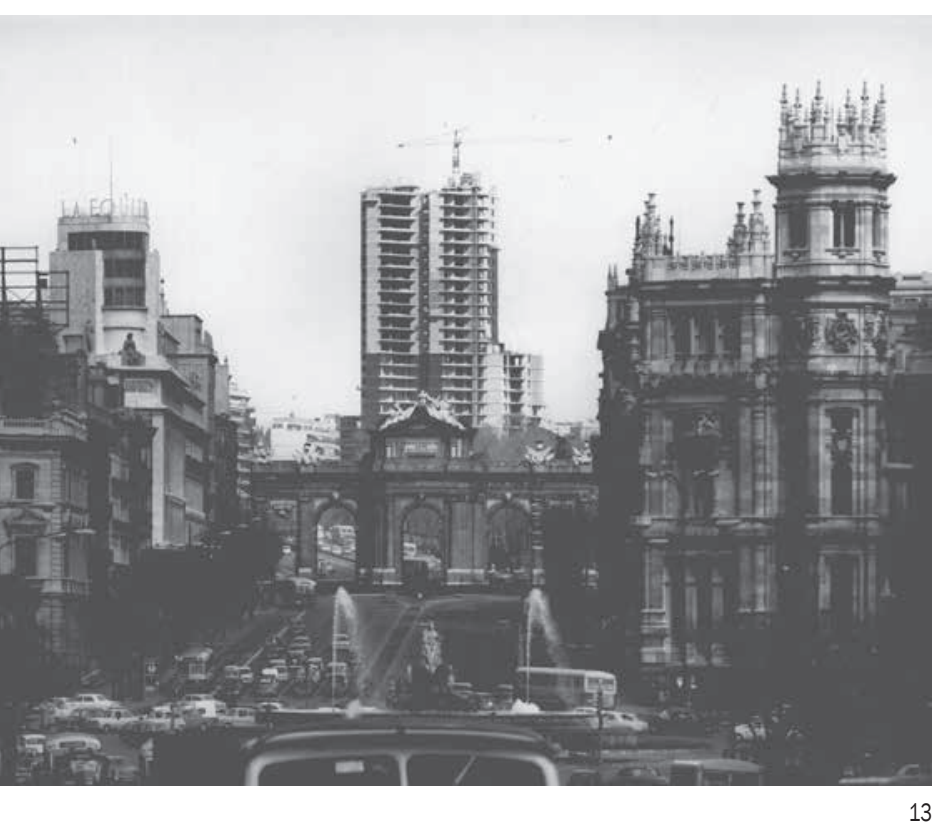

lejanas sobre el Parque del Retiro devolviendo al morador le confirieron la seguridad necesaria para poder culminar la intimicady la expansion, la penumbray la luz. El arqui-- una obra que, por razones ajenas al proyecto, estuvo a tecto resuelve con elegancia y una aparente facilidad la articulacion de los distintos volumenes en un ejercicio de extrema dificultad tecnica y programática

Las innovaciones tecnicas del proyecto atectaron a las instalaciones del edificio, con control electrónico de temperatura en el sistema de calefacción y servicios individuales de acondicionamiento de aire, empleados por primera vez en un edificio residencial, asi como vidrios Termophane o Parsol en las zonas de comunicación. Esta voluntad de responder a las exigencias y demandas de momento acercandose a los parámetros constructivos de sociedades más desarrolladas que él bien conocía punto de ser no ser concluida $a^{34}$

La polémica, recogida en la prensa de la época, singularmente en el diario $\mathrm{ABC}^{35}$, conviritio al proyecto en motivo de supuesto "escandalo". Carvajal respondería ponderadamente que hubiera sido justo no hablar de "escándalo", sino de polémica, porque esto es lo único que existe, opiniones y sólo encontradas opiniones sobre un tema de estética opinable"36 (figura 13).

En una medida y elegante respuesta en las páginas de ABC Carvajal agradece las discrepancias fundamenla historia por las que aprende, como tantas veces repetiría a sus alumnos

33. Ficha técrica del proyecto. Solar: 2.802,70 m; Atturas: 28 sobre rasante y cinco bajo rasante; Volumen construido: $75.534,50 \mathrm{~m} 3$; 0cupación del solar:

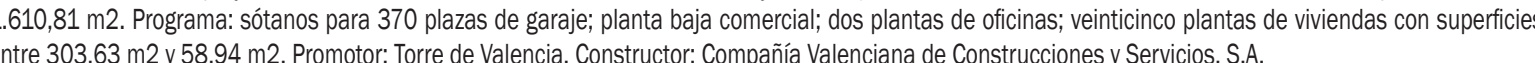
34. "En este mes los vecinos afectados por la construcción de la Torre de Valencia retiran su recurso que, atendido por el Ministerio de Vivienda, había parallzado las obras del discutido edificiio. objetto de clamorossa polémica y acusada de estropear l a airosa perspectiva de la Puerta de Alcalá, de lo que con aguda intención se hace ecco el lápiz de Mingóte, la torre de Valencia superó el peligroro de la demolición que muchos querían" en Diario ABC, Blanco y Negrón. Madrid

35. El Diario ABC, el 11 de abril de 1971, pp. 122-127, bajo el titulo “Encuesta sobre un gran escándalo" recoge las opiniones sobre la relación espacial entre

36. Carvajal Ferrer, Javier: Diario ABC. Madrid: 27 de abril de 1971, p. 40
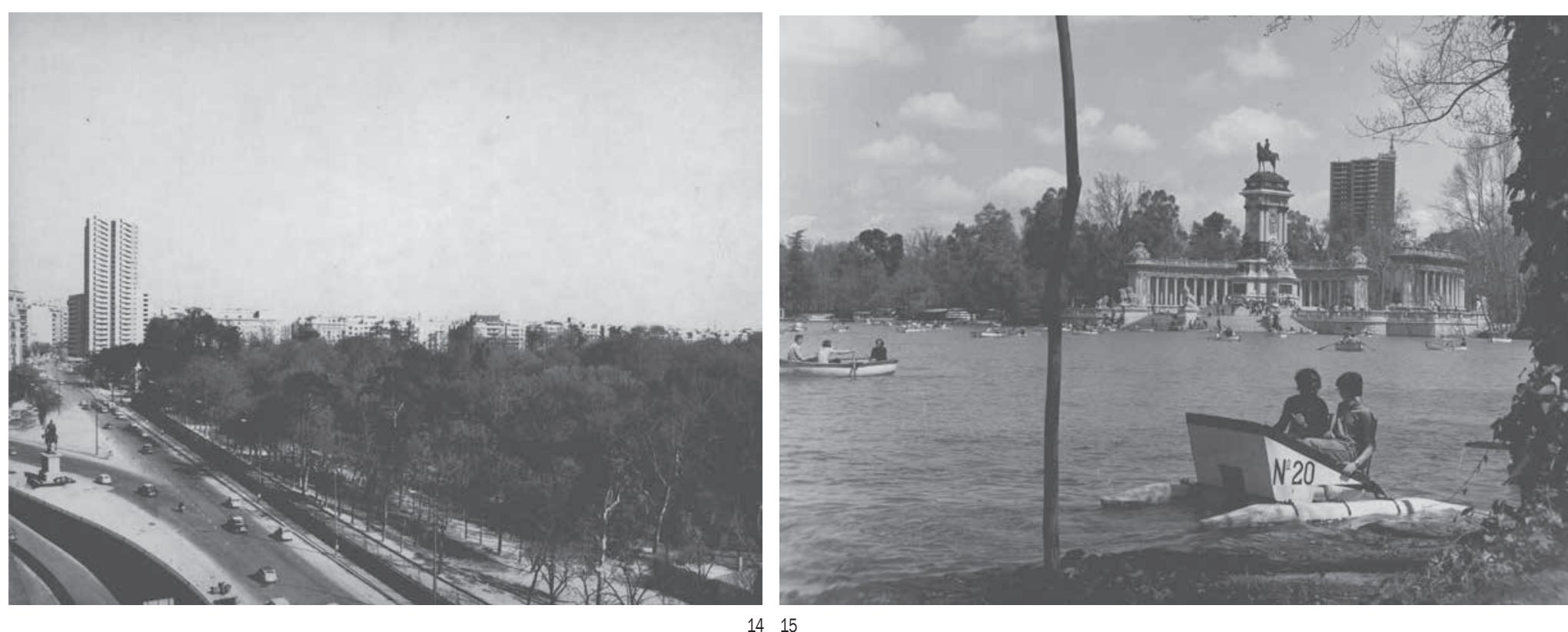

que las distintas arquitecturas, a lo largo de las sucesivas generaciones, se sustliuyen, conviven y se solapan. Pero ello solo es posible desde la calidad, seguridad determinación de un proyecto que garantice su vigencia. No deja de ser una feliz coincidencia que la publicidad aparecida a toda página con una fantastica imagen de la Torre en el periódico ABC (viernes 2 de abril de 1971) bajo el titulo "Torre de Valencia. su retiro particular", indicase como un molivo de calidad y garantia: "Arquitecto: Javier Carvajal, autor del Pabellón de España en la Fería de Nueva York" (figura 14).

LA LECCIÓN DEL PROYECTO: VOLUNTAD DE SERVICIO Y FIDELIDAD AL TIEMPO PROPIO En efecto, ambos proyectos participan de la voluntad de contribuir, desde el tiempo histórico propio, a la evolución de la arquitectura. Carvajal fue un acerrimo defensor de la formación humanistica del arquitecto y del sentido histórico de su quehacer como "eficacia significante". Cabe reproducir la respuesta que daba en una entrevista precisamente en este sentido:

La Arquitectura debe entenderse por eso como respuesta a necesidades materiales (que configuran su eficacia de uso); pero también a la emoción y a la belleza, que le confieren su condición significante. Cualquier planteamiento que disocie la teoria de la realidad, lo pánico y lo angelico, y que no tenga como centro al hombre total que vive de sueños y realidades, de certezas y misterio, no es propio de la Arquilectura, que siempre ha contemplado ef hombre completo, configurando para él los espacios. (...) La posmodernidad, que pretende presentar como modelo el reduccionismo arquitectónico y el caos como unica salida a la crisis de la modernidad, olvida que la historia de la cultura no es sino la historia del orden frente al caos; y que el caos, tal como se presenta, supone la negación del complejo orden que la Arquitectura construye al semcio del hombre complejo, y no del hombre reduccionista, solo atento a la materia mensurable y a la economia, como unica medida. (...) Cuando la Arquitectura recobre su len guaje y estructura de servicio, será valorada como tal, y recuperara su papel de signo de la sociedad, la misma que hoy se desentiende de ella" ${ }^{37}$ (figura 15).

37. Vicens Hualde, Ignacio; Llano Sánchez, Rafael: "Entrevista a Javier Carvajal: La Arquitectura debe recuperar el misterio". En: Nueva Revista, 58. Madrí 1998. 
Acaso esta voluntad de servicio y permanencia, nutrida en la exigencia de la calidad del proyecto desde la delidad al propio tiempo, tantas veces evocada por CarFajal a sus alumion, constituya su lección más duradera. Fidelidad que requiere de las enseñanzas de la

Esta vigencia requiere del concurso de la materia. anteriormente hemos analizado la determinación constructiva del proyecto, es necesario recordar también la importancia que para Carvajal tiene la incorporación Porque en él la materialidad trasciende su condición física para sumarse al esfuerzo de contribuir a la evolyción de la arcuitectura desce la afimación de la conemporaneidad Para el verdadero arquitecto, una vez conquistados los requermientos técoicos -algo que un buen profesiona debe dominar-, su voluntad de expresón y permanencia le impusan a un objetivo esencia: ser en el tiempo $Y$ como decimos, es en este proceso en el que Carviu concede a la materia su lugar privie-

permanencia. Esta fecunda relación entre estructura, materia e imagen encuentra en la Torre de Valencia una alzmno sabion. Por ello insistia tan tenazmente a sus crear la realidad unitaria de la obracon encaminado a

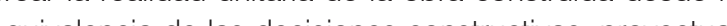

Las lecciones destiladas en este texto se engloban del arquitecto, a su voluntad Más allá de la con actu entre docencia y praxis, de la precisión de sus dibujos de las razones técnicas y espaciles de sus obras de lirmeza de sus conviciones ante las adversidades, nos queda como lección más preciosa y exigente, a voluntad de fundir imaginación y realiad con lánica herramienta de la bondad del proyecto arutectónico: Para mísoñ es super has mezquinas cotsos delintés más inmediato y ruin Para míesta aclitud compota imaginar a realidad como sila bondad de nuestros proyectos no tuviera más límites que nuestra voluntad y luego cons- truir su realidad como si de nada valieran los sueños" 38 .

\section{Bibliografí:}

A..W.: J. Carvajal. Arquitecto. Madrid: Servicio de Publicaciones del COAM. 1991.

AA.W.: De Roma a Nueva York: itinerarios de la nueva arquitectura española 1950-1965. Acto académico de la Universidad de Navarra a Javier

Hernández León, Juan Miguel (coord.). Libro Blanco. Titulo de Grado en Arquitectura. Agencia Nacional de Evaluación de la Calidad y Acreditación.

Carvajal Ferrer, Javier: Sobre la génesis del proyecto. Pamplona: T6) Ediciones. 1997.

Carvajal Ferrer, Javier: Curso abierto. Lecciones de arquitectura para arquitectos y no arquitectos. Madrid: Servicio de Publicaciones. Colegio oficial de Arquitectos de Madrid. 1997.

Carvajal Ferrer, Javier: Javier Carvajal. Madrid: Editorial Munilla-Lería. 2000.

Delgado Orusco. Eduardo: Porgue vivir es dificill: conversaciones con Jvier Carvajal. Madrid: Universidad Camilo José Cela. 2000.

Diario ABC: Homenaje al arquitecto Javier Carvajal. Madrid: 19 de diciembre 1964. p.97.

Diario ABC: Encuesta sobre un sran escándalo. Madrid, 11 de abril de 1971, pp. 122-127.

Diario ABC: Madrid: 27 de abril de 1971, p. 40 .

Diario ABC: Blanco y Negro. Madrid 4 de diciembre de 1971, p. 109.

Martín Larumbe, Celia; Chocaro Bujada, Carlos (coord. exposición): Loewe años 60. Cuestion de estilo. Pamplona: Universidad de Navarra, 2008 Pozo Municio, José Manuel (ed.): Las exposiciones de arquitectura y la arquitectura de las exposiciones. La arquitectura española y las exposiciones

Pozo Municio, José Manuel (ed.): 1964/65 New York World's Fair, The Spanish Pavilion. Pamplona: T6) Ediciones, 2014

Vicens, I.: Llano, R. "Entrevista a Javier Carvajal: La Arquitectura debe recuperar el misterio". En: Nueva Revista, 58. Madrid: 1998.
Carlos Labarta Aizpún (Zaragoza, 1962). Arquitecto, ETSA Universidad de Navarra, 15/05/1987. Becado Fulbright y Master GSD Harvard Univesity, 1990. Doctor arquitecto Universidad de Navarra 6/10/2000, con Premio Extraordinario. Profeseror Titular de Proyectos Arquitectónicos desde 18/10/2004, actualmente en la EINA Universidad de Zaragozza yen la ETSAUN como Profesor Vis tante. Articulos en revistas como Ra 3 y 14, 1999 y 2012, Tectónica 15, 2003 , y en libros como Architects' Journeys/Los viajes de los

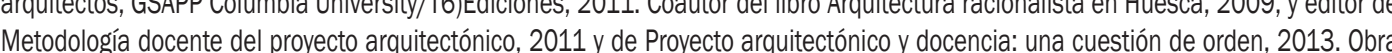
incluida en exposiciones conjuntas como Habitar el Presente, Nuevos Ministerios, Madrid, 2007. Premio García Mercadal, COAA, 2008 y Prefinalista Bienal de Arquitectura Española, 2013 .

Jorge Tárrago Mingo (Burgos, 1975). Arquitecto, ETSA Universidad de Navarra (2000). Doctor arquitecto por la ETSA de la Uni versidad de Navarra (2005). Profesor Titular de Proyectos (2014) y actualmente Director del Departamento de Proyectos, Urbanismo, Teoría e Historia. Es autor de los libros Habitar la inspiracion/construir el mito. Casas-taller de artistas en el periodo de entreguerrias (Valencia, 2007) y co-autor de otros como La maison de l'artiste (Rennes, 2007), Iglesia y Convento de Santa María de Belén. Stella Maris, José María García de Paredes 1961-1965 (Pamplona, 2011), Architects' Juurneys (New York, 2011). Coordinador de la revista 180, Bauwelt o ARQ entre otras. 


\section{CARVAJAL Y LA VOLUNTAD DE SER ARQUITECTO: LA CONSTRUCCIÓN DEL}

\section{CARVAJAL AND THE WILL TO BEAZ}

AND EFFECTIVE BEAUTY

Carlos Labarta Aizpún; Jorge Tárrago Mingo

p.39 DREAM AND REALTY: AREINTERPRETATION OF THE MODERN LEGACY

It seems appropriate to refer to the figure of Javier Carvajal Ferrer (Barcelona, 1926-Madrid, 2013) as one of the architects and professors who, from the sixties, helped to shape the professional and educational map of Spanish architecture. In his case
the paths of architect and professor run intertwined to the point of not being able to dissociate one from the other: converging vocations lived with overflowing generosity and passion. In his later years as a teacher he used to say to his closest friends that his best work-and that he was most proud of-were his students. 'As a notable group of prestigious scholars in charge of chairs and teaching responsibility positions in the most diverse schools of our country, they seek to emulate the consistency of their master the Valencia Tower. Madrid (1970-73)-are extended both in his works and in his students and, somehow in the sudents of his students (Figure 1 )

Teaching and practice are linked by Carvajal through the decisive preponderance given to the architectural project to whose effective construction-from emotion and technique, from the dream and reality-devoted his efforts. The architectural project is to Carvajal the specific field of the professor's research, and at the same time the mechaniism by which the practitioner mus creative ideation and in tactual construction. Architecture is not a drawn art, but a buitt art" 2 That desire for authenticity resulted in extreme dedication devoted to every project he undertook

This text aims, in light of these two flagship projects, to evoke simultaneously, both the virtues of his architectural work and the lessons learnt in his teaching. For this we turn to the rigor and beauty of the plans that he drew and that turn the project into a the most controversial With apparenty different luck and recognition both projects are equally examples under the perspective the most controversial. With apparently different luck and recognition, both projects are equally examples under the perspective
of the project as a mechanism to achieve effective beauty. And both translate similarly-one from the inner satisfaction produced

p.40 by praise, the other from the fortress facing falsehoods-the profound vocation of the architect to shape his dreams through the project. Both works illustrate how firm confidence in the quality of the project remains as the best contribution to the evolution a

"Cream of the possible". So Carvajal used to begin and end his endless sessions of project corrections in the studios of
architecture
the schools of architecture, mainly in Madrid and Pamplona, to which he devoted most of his time. His long and fruitul teaching career ${ }^{4}$ achieved early success, obtaining after his graduation. This achievement will always be remembered as being the first in the School of Madrid by an architect who

operated in the area of modern architecture.
His tranining, as it happened to his generation, was marked by civil war and years of hardship and isolation, followed on the
contrany by academic orthodoxy and certain contempt for what was done outside except for the teaching of some professors.

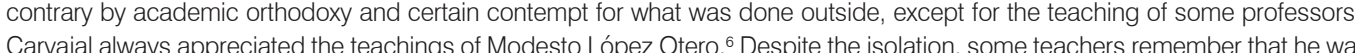
the only student who, at that time, consulted foreign journals.? Shortly afterwards Carvajal would become, following Fullaondo's "rationalist ascending" and "organic-expressive inflection", a generation that had found its way facing and battling "countless

Obtaining the architectural project chair coincided with the construction of the pavilion that would represent Spain at the the profession and teaching Carajal's poincoidence proves the dimension of his intense and simultaneous dedication both to national and international criticss,' and found in this early work recognitition, more elusiviv than those of his generation old he triumphed in New York obtaining the Gold Medal against competitors such as Philip Johnson and Kevin Roche with the Pallion of the City of New York and the IBM Pavilion, respectively.

.41 innovation and the desire to incorporte in his archititecture consd idea emerges from a deep knowledge of reality. Technica greatest interest. With the Spanish Pavilion at the World's Fair in New York' possibilities of precast concrete facade elements. In the following decade he would culminate this research in the Valencia Tower "Undoubtedly, this building is one of the buildings that I feel more satisfied with of all I have built. The Tower is fine, in my opinion,
strong clear with adequate plans and a good image I Iesigned the Tower and the Tower is still there, I defended the Tower the Tower remains".

To understand these two works, as well as the architect's teaching, we must refer to the sources where the young Carvaja studied as he describes them and whose ideas and principles he learnt: "But we were dazzled, as students and young architects of that moment, with the work of the great masters: Gropius, Le Corbusier, Mies, Aatto, Neutra, Saarinen, Terragni, Breuer, and many Rome between 1955 and 1957 would contribute decisively to his knowledge of these masters And, with them Canaial would also refer to closer masters whose teachings are equally present in these early works: "Our young masters then, almost of our own age, were Coderch, Sota, Sáenz de Oiza, Fisac, Cabrero, Aburto y Blanco Soler, Sert, Aizpurua, Candella and the whole series, already

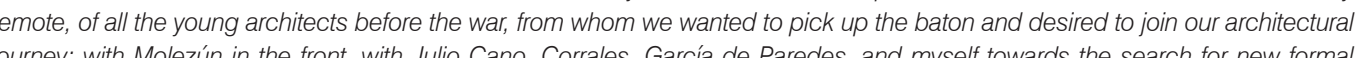
expressions within the line of the modern movement that we felt as our own, that touched us with more strength and commitment than the old inetorical styles or the historical examples that could move, and actualy do move, to admiration but not to repettition, because their circumstantial reality did not show us any similarity with our realit'

These two projects mark respectively the beginning and hie end of the second stage of his career, 14 perhaps the most genuine in which the Somosaguas houses, already part of the history of contemporary Spanish architecture, also belong. 15 The project of the tower his tath and trustin the goodness of architecture as an antidote to all sort of contingenciess, subject to trends or occasional criticism. The Valencia Tower represents for him the most demanding test and, with it, his lesson least understood. The desire to achieve effective beauty, away from cyclical topics, simultaneously rooted in tradition and open to modernity he had

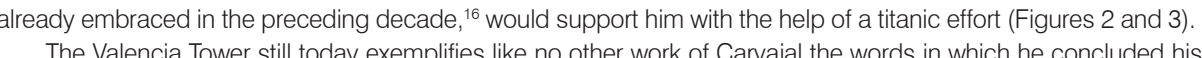

The Valencia Tower still today exemphiles A desire for permanence, overcoming the keys of time, that starts from the only tools at his disposal: drawing as language and expression of the architect.

THE PROJECT AS A MECHANISM OF EFFECTIVENESS: COMMENTED DRAWINGS AND THE ABSTRACTION OF THE

The project for Carvajal is never an end in itself, which may tend-as so often occurs-towards complacency. The project is not othing more than a mechanism for and beauty, but also it is constituted as the mechanism for them: "Because ine project is specilic"."' Similarly, the drawing is seen as the tool serving this purpose

A review of the Pavilion original drawings warns us of the importance Carvajal gives to the graphic representation of his
projects and also translates to his teaching. A tireless drattsman, he was able, as Campo Baeza states, to "add dimensions to the air"'1" in his belief that drawing shelters all the information and the potentiality of the built work The drawing of the plans is dated June 1, 1963, and on the cover both the architect Carvajal and the drattsman G. Hoyo are listed, as unequivocal proof of

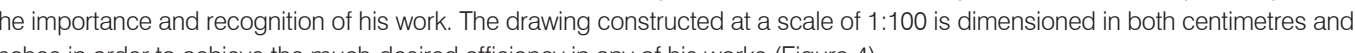
nches in order to achieve the much-desired efficiency in any of his works (Figure 4)

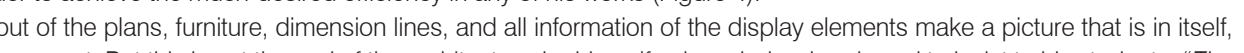
a valuable document. But this is not the end of the architect, as he himself acknowledged and used to insist to his students: "The
lines that make the plans have no artistic value in themselves, nor is its purpose to have it. but to be an abstract expression of the uture reality they represent". 19

The richness that contributes to the construction of the plan, the arrangement of furniture, and the different definitions of material or display elements do not cloud but strengthen the abstraction of an inherited composition of the tenets of modernity that
Carvaial embraces in this work without living up to the deepest roots of Spanish architectural tradition either culturec or popular Thus, straight lines, squares, rectangles and circles, all basic geometric shapes are arranged-with different sizes according to the needs they serve - "disorderly". According to the compositional criteria they reformulate new relations of order that no longer have to do with hierarchy but with classification, that do not refer to equality but to equivilence. Any of the fragments of the plans can be read in this light. Carvajal's master teaching resonates in the perfect composition of each of them-and, from them, the (n)

of the dof of th the complexty of the architectural fact and of its unity. It is stimulating to observe and patienty sit through each on a dotals ol heration that Carvaial dimensions and anticipate the tour of visitors, checking virtuosity, including: "vevet seats without backs", "a monk by Zurbaran (the church)", "wooden plattorm", "timber piece", "clay vessels", "trailero chair", and "the Countess of Chinchón by Goya (the nobility)". All of this is properly intermixed and arranged in construction axes. This is reflected, among other information, in the plan of the entrance floor to the Exhibition Hall .No. 8 fFigure 5). And thus this strategy is repeated in each and everyone of the projected spaces.

Thus, the architect skififly handled the elements that turned the space into a fantastic and exotic place: the visitor was confronted with a complex but peaceful atmosphere, with strong light and careful darkness, with spatial sequences around a courtyard, with diagonal views and broad perspectives. The fabulous amount of objects and pieces of art were displayed
sometimes integrated into the pavilion architecture-bars, murals, stained glass windows, sculptures and lattices specifically commissioned by artists for this occasion-and other times on tables, showcases, or on the flloor 20 in short, an opulent space, 
where it was difficult to distinguish what was and what was not exhibition. Carvajal had arready used similar project mechanisms showcases, as well as gathered on tables or in structures on the floor, which together with design furniture and textiles saturated

reeated many times by him -that "architecture is an art with a roasonation as diverse as this, he claims-in his final definition reflects and expresses abstractly what the architect already knows specifically.

Such is the condensation of information that drawing in its definition approaches constructed reality. Therefore, Carvaja

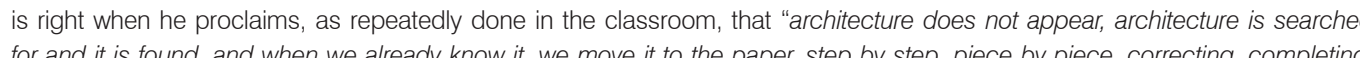

p.45 adding the integral parts of a whole" 22 In this way the drawings of the pavilion plans are also built. Piece by piece, cinpt to jing with a complete view of the architect's work from the very beginning: "know how the work will evolve, even in its smallest details including turnishings, but it's hard for me to translate my intentions and emotions".".

Carvajal teaches that one who can draw well, that is with graphic efficiency and technical precision, will be able to build properly. His projects shelter the potential of the built work as the ultimate and only aim of architecture

THE CONSTRUCTIVE AND SPATIAL DETERMINATION OF ARCHITECTURAL DRAWING: THE VISUAL ORDER OF MATERIALS From the vast collection of drawings, it is worth analysing a few of them where ideation, development, and construction of the project are exemplified.24 Under the movement of the floor plan the module appears as a mechanism of order. The steel columns are arranged in a grid of ten by ten metres $(32$ feet $93 \%$ inches) also used by the architect in a former proposal for the lesser known
1956 Venice Biennale Pavilion.2. In both cases, this decision implies a functional reason: to obtain the required openness in The predominantly used profiles were WF (Wide Flange) for any vertical element and S (Standard) for holzc

ccording to the American standard which is the more similar to the European HEB and IPE. Steel decks thereby achiever relatively short construction deadlines, which resolved the slabs. The plan 63-74-23, A-11 "Pre-cast reinforced concrete elements for exierior walls" describes how the metal frame on the top loor was covered with $7 \mathrm{~cm}$ thick pretab concrete pieces anchored

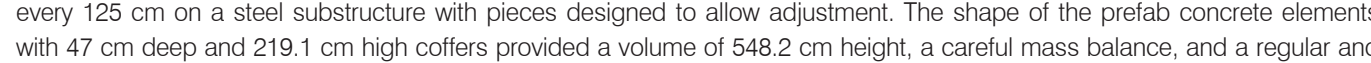
projected by the slab borders, $40 \mathrm{~cm}$ exactly, to accentuate a continuous shadow line and lighten the weight mass perception (Figure 6). Carvajal subordinates constructive decisions to visual interest

Indeed, the pavilion could look discreet, hermetic, and contradictory because of its whitewashed walls on the ground floor the thens a walls that run and close the entrance floor (Figure 7).

The floor plan was not a horizontal plane but a topography according to the physical characteristics of the site and to the various stresses that the architect created according to the programmatic and spatial demands (Figure 8)
This reality is evident to the extent that its exterior soberness did nat permis the viewer to

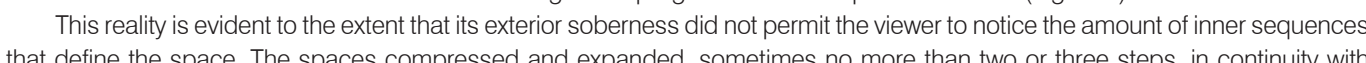
that define the space. The spaces compressed and expanded, sometimes no more than two or three steps, in continuty with

p.46 different areas and dissolving container and content. This strategy somehow emulated the Moorish architecture of the Alhambra in Granada he admired, and to which he referred frequently and visited countless times with his students

Both the ceilings of Moorish influences, formed by a coffer based on square pieces of dark walnut wood and the lighting were

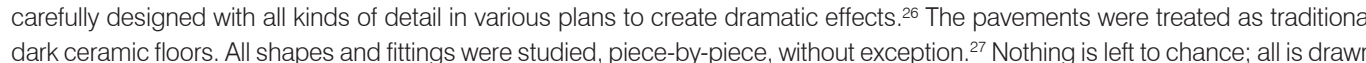
(Figure 9).

Carvajal acknowledged "tradition as a starting point, as fidelity to the present time". He recognized that he was a 'resonator of the situation of a country through the practice of architecture, maybe not at the level of technical development that he could find in New York, but of a country with a strong culture "in motion and towards the future",28 The formal and technical research with
pre-cast concrete still had to overcome the reluctance of Mies van der Rohe. Carvaial, who left self-governed Spain participted "I can give you some advice. If you want to succeed with your Pavilion do not use seriation or prefabrication". Carvajal replied he could expect that advice from any other architect. And Mies replied." On the contiay, lam the only one who can give It. I was the inventor of those things and see (he pointed with a sweep of his hand to the panorama of Fifth Avenue), what they have done with TECHNICAL COMPETENCE VERSUS EVENTUALITY: THE PROJECT AS AN ALLY

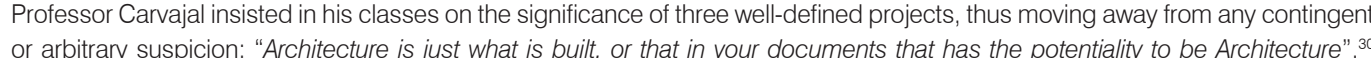
p.47 Carvajal achieved higher technnical qualifications than his generational colleagueses, often surprisised by the degree of perfection in Carvajal achieved higher technical qualifications than his generational colleagues, often surprised by the degree of perfection in
his work.1 The qualification reached in New York continued in the Valencia Tower, whose thirty-three floors (twenty-eight above ground flloor) determined a vertical structural solution of a mixed solution of metal pillars and reinforced concrete core bracing. The horizonlal struture was made with bidirectional-ribbed slabs. The desire for the whole project to be made with a single material-reintorced conciete in any of the possibitities from its construction processes-led the architect to continue his research with prefabricated panels, successfully tested in the pailon

Along with plans, he used models-as he used to require of his students-and even tests on photographs of the building
process as verfification mechanisms of a hardly imaginable solution only attending to the previous state of the site The desire to sculpt a tower in the air made Carvajal verify the formal result trom the beginning of the project. The images of the model reveal the authenticity of an architecture defined by the movement of shadows (Figure 10). No doubt the importance of the project and the controversy in which it was involved led the architect to find the most satisfactory solution. The slender shape of the tower
under construction emerges from the base of the two office floors thus solving the tension of the connection between vertical and horizontal planes by an access floor that frees up 50 per cent of the site (Figure 11).

Not only had the use of prefabricated systems related both projects ${ }^{32}-$ both in the horizontal base of the pavilion and in the vertical construction of the tower-Carvajal tested similar strategies in past projects influenced by 'close masters' such as Coderch. The spatial research made in the pavilion was reinterpreted a few years later in the tower flloor plans, including courtyards
in the air and proposing 'single-family houses in height' (Figure 12). Each house has a separate access and distant views over the Parcue del Retiro returning the inhabitants privacy and expansion, darkness and light The architect solved with legance and $p$. ability the articulation of various volumes in an exercise of extreme technical and programmatic difficulties. ${ }^{38}$

Technical innovations such as electronic temperature control in the heating system and individual air conditioning were first used in a residential building, also Parsol or Termophane glass in common areas. This desire to meet the needs and demands of

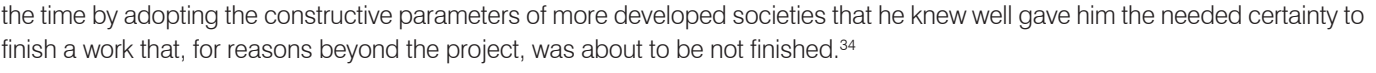
The controversy, portrayed in the press of the time, particularly in the ABC, 35 resulted in a kind of "scandal". Carvajal ponderously responded "it had been fair not to speak of 'scandal" but of controversy, because this is all there is, only opinions and conflicting opinions on a debatable topic of aestheteic" 36 (Figure 13).

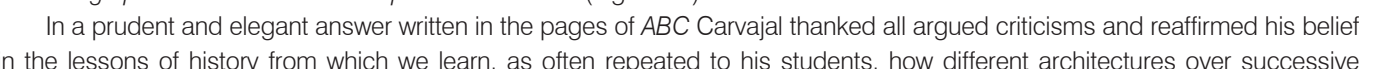
in the lessons of history from which we learn, as offen repeated to his students, how different architectures over successive
generataions change, coexist, and overlap. But this is only possible from the quality, conviction, and determination of a project to ensure its validity. It is not a happy coincidence that a full-page advertisement with a fantastic image of the tower appeared in the
$A B C$ (Friday April 2 , 1971) entitled "Torre de Valencia: your private retreat", indicated as a sure reason for quality: "Architect: Javier Carvajal, author of the Spanish Pavilion at the New York Fair" (Figure 14).

THE PROJECT LESSON: THE DESIRE OF SERVICE AND FIDELITY TO ONE'S OWN TIME

Indeed, both projects involved the desire to contribute, in one's own time, to the evolution of architecture. Carvajal was an ardent
defender of the humanistic education of architects and of the historical significance of his work as "significant efficacy". It is worth

reproducing the answer he gave in an interview in this sense:
"Thereforore, Architecture should be understood as a response to material needs (that shape architectural efficiency in terms of

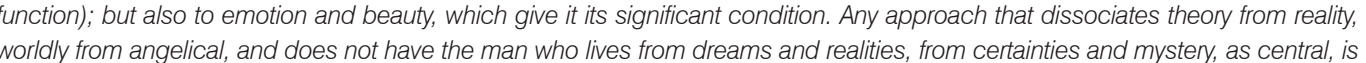
not proper for Architecture, which has always considered this total man and has configured spaces for him. (...) Postmodernism. which aims to present a model of architectural reductionism and chaos as the only solution to the crisis of modermity, forgets that the history of culture is the history of order against chaos; and that chaos, as it is presented, represents the lack of complex order

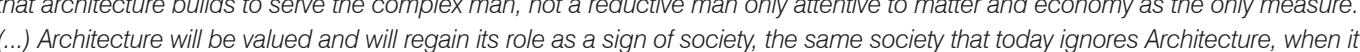

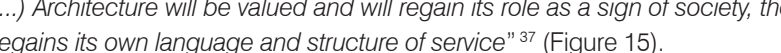

Perhaps this desire tor service and permanence, nourished by the project requirement of quality and fidelity to its own time, $p .50$ so often evoked by Carvajal to his students, constitutes his most lasting lesson. As Carvajal argues, fidelity requires the lessons of history, including modern history to dream of the future.

This requires participation of matter. If we have previously analysed the importance of the construction of details, we
should also remember the importance that Canaial gave to the incorporation of matter from the initial stage of design For him materiality transcends its physical condition to inin the effort to contribute to the evolution of architecture trom the assertion of its contemporaneity. The true architect's essential goal, once the technical requirements are achieved, which is something that a good professional has the obbligation to control, is to be in his or her time. And in this process is where Carvajal gives matter its prominent role from the beginning of the design, fusing genesis and permanence. This fruititul relationship between structure, a decisions.

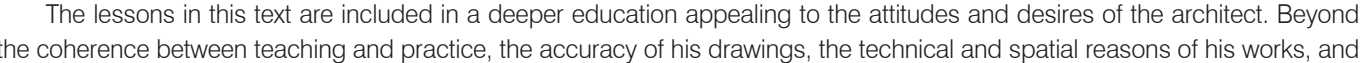
the coherence between teaching and practice, the accuracy of his drawings, the technical and spatial reasons of his works, and
the strength of his convictions in adversity we have the will of imagination and reality fused with the goodness of the architectural the strength of his convictions in adversity, we have the will of imagination and really yused with the goodness of the architectural 

and then build their reahly as if dreams were worthless."

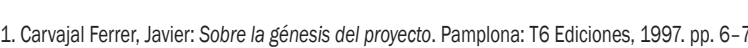

2. bidi.

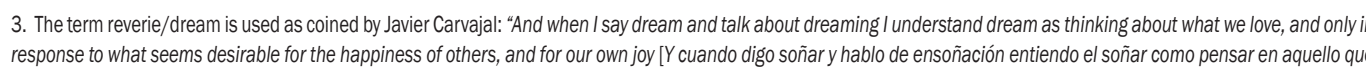

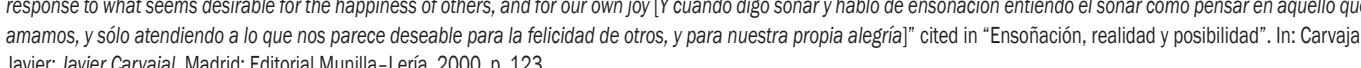

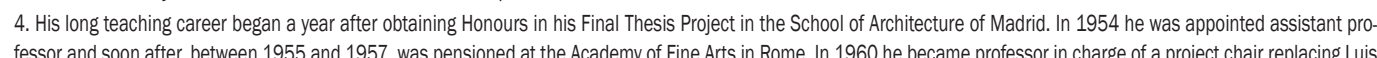

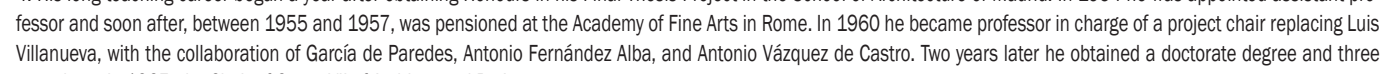

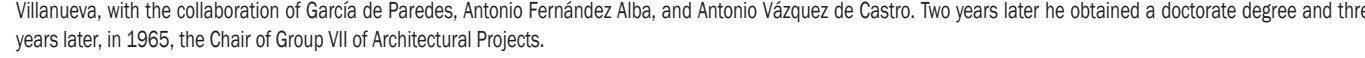

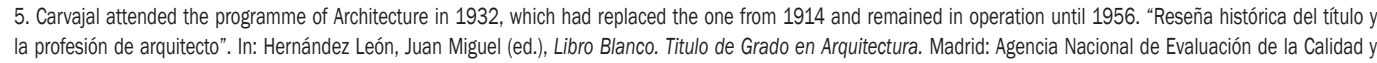

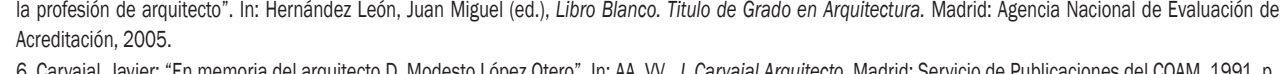

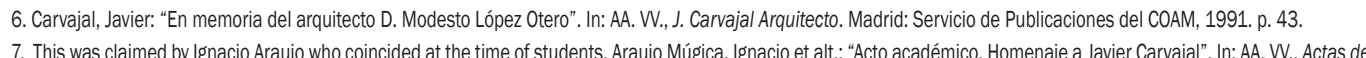

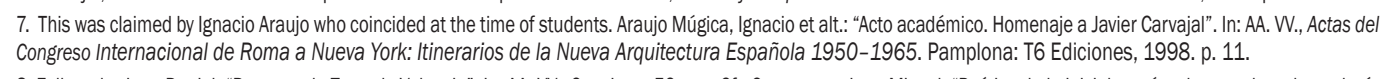

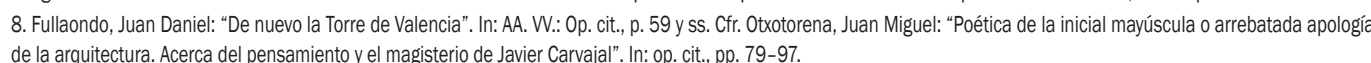

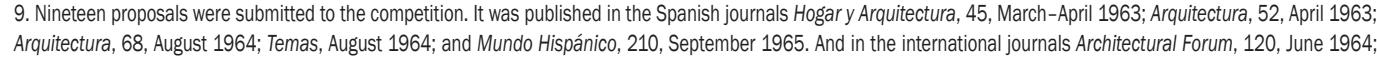

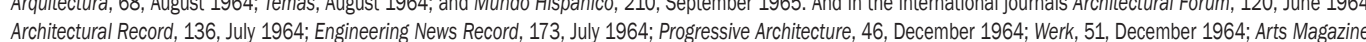

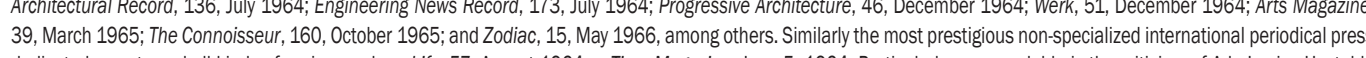

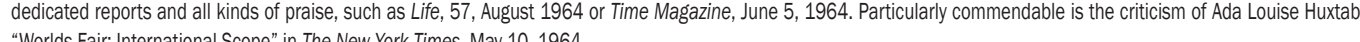

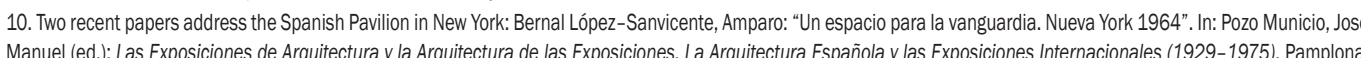

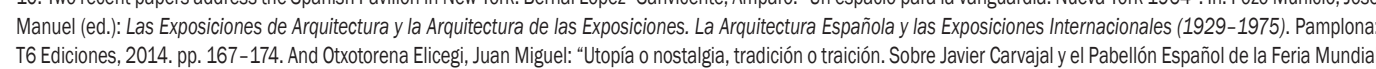

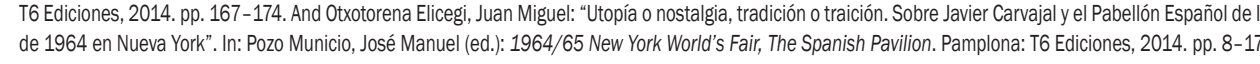

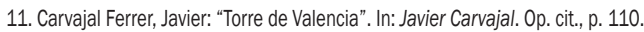

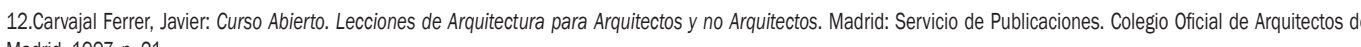
Madri, 1997. . . 2.

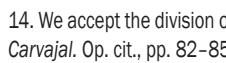

15. The houses Casa Carvajal(1964-66) and García-Valdecasas (1964-66), both in Somossaguas, Madrid, also belong tot this stage

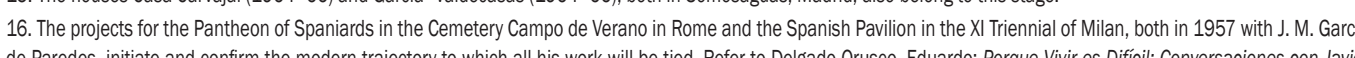
Vivires Dificil: Conversaciones con Javier 17. Cavajal Ferer, Javier. Op. citi, p. 105.

18. Campo Baeza, Aberto. Op. Cott, p. 82

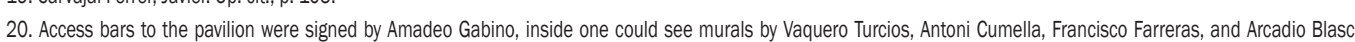

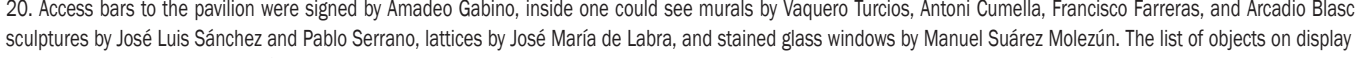

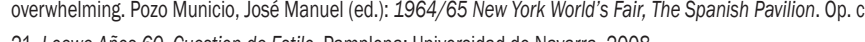

21. Leeve Años 60. Cuestion de Estili.

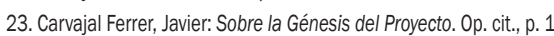

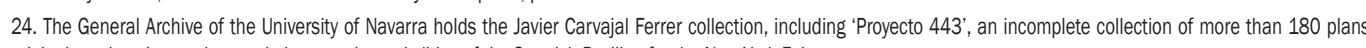

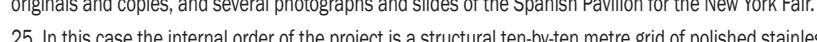

25. In his case the men

28. Diario ABC: Homenaje al arguitecto Javier Canajali. Madrid: December 19, 1964. p.97.

29. Carvajal Ferere, Javier. 0p. cit, p. 25.

Cénessis del Proyecto. 0p. cit, p. .7.

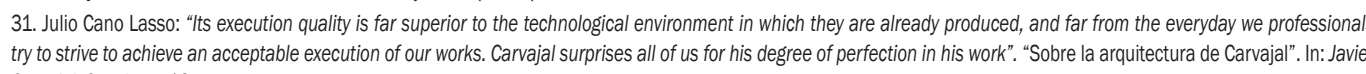
Carvajil. op. citi, p. 12.

32. Together with the spatial research of the pavilion courtyards, it is woth remembering the complex articulation of the walls of the House Canajal and the House Garcia Valdecasas (Somosaguas, Madrid, 1964-66).

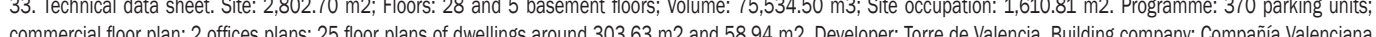

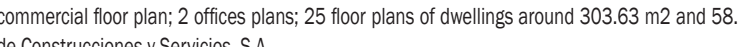

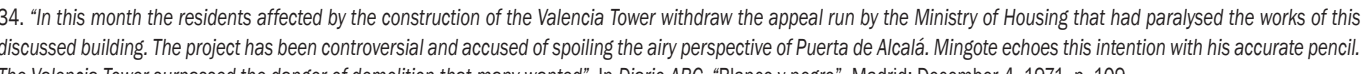

35. Diario ABC, April 11, 1971, pp. 122-127, "Encuusta sobre un gran escandala" collects different opinions about the spatial relationship between the Valencia Tower and Puerta de Alca

36. Canajall Ferere, avier: Diario ABC. Madrid: April 27, 1971, p. 40.

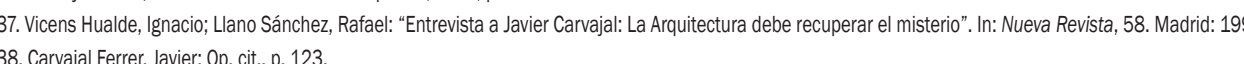




\section{Autor imagen y fuente bibliográfica de procedencia}

Información facilitada por los autores de los artículos: paǵina 20, 1 (Fermández-Allba, Antonio: El Observatorio Astronómico de Madrid. Juan de Villanueva, Arquitecto.

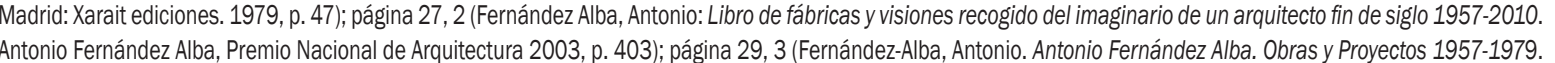

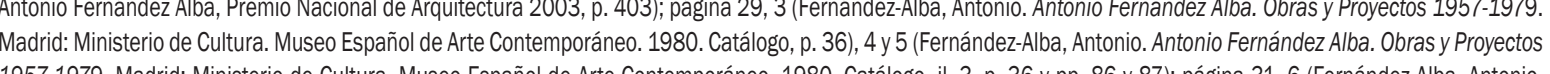
1957-1979. Madrid: : Ministerio de Cultura. Museo Español de Arte Contemporáneo. 1980. Catálogo, il. 3, p. 36 y yp. 86 y 87); página 31, 6 (Fermández-Alla, Antonio,

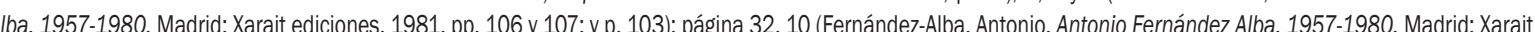
ediciones. 1981, p. 54 ), 11 (Femández-Alba, Antonio. Antonio Femández Alba. 1957-1980. Maddrid: Xarait ediciones. 1981, p. 76); página 33, 12 (Fernández-Alba, Antonio. Antonio Fernandezz Alba. 1957-1980. Madrid: Xarait ediciones. 1981, p. 71), 13 (Femandez-Alba, Antonio, El Observatoroio Astronómico de Madrid. Juan de

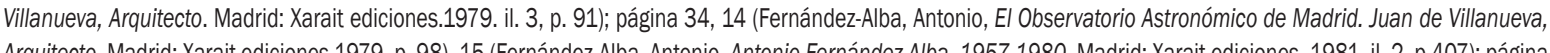

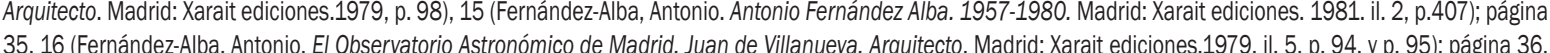

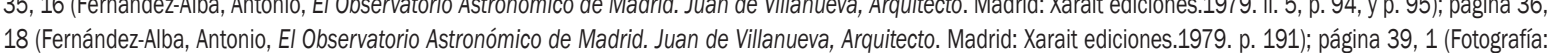

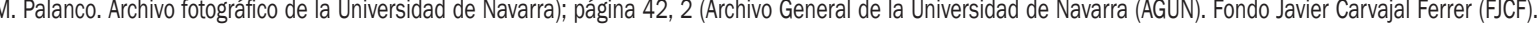

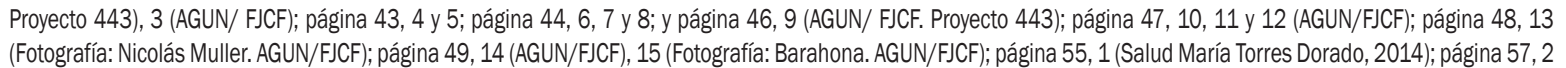

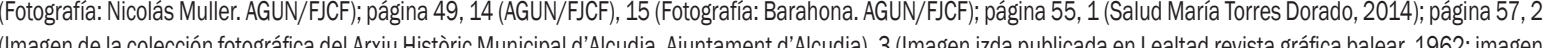

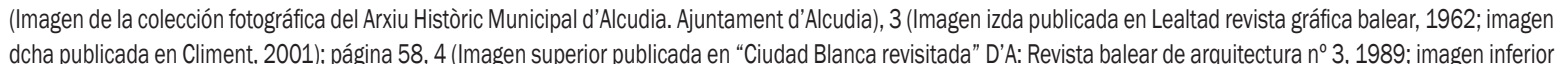

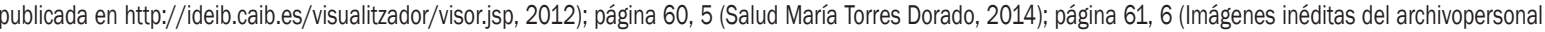

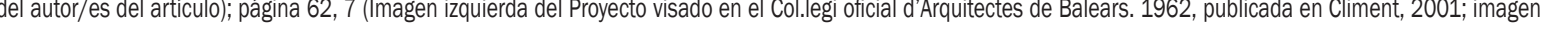

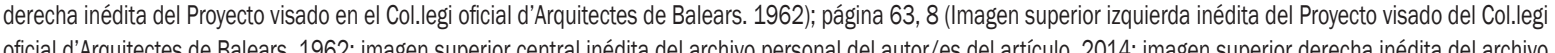

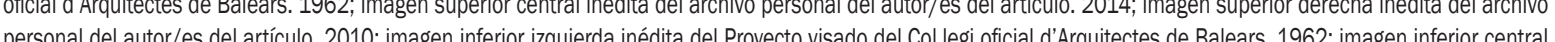

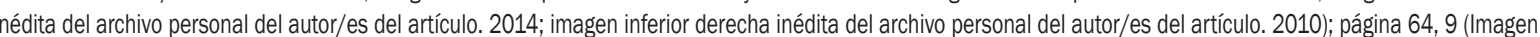

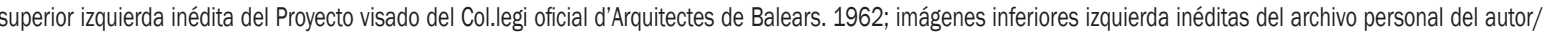

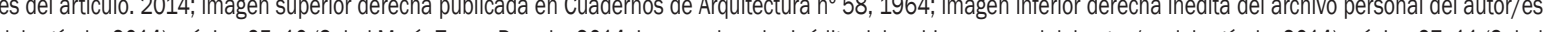

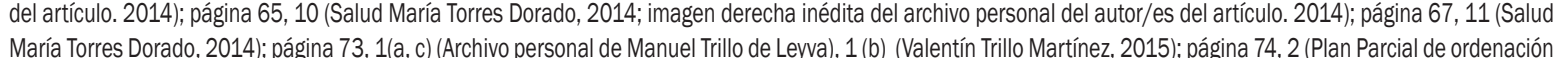

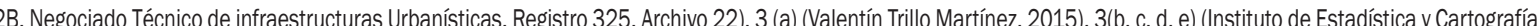

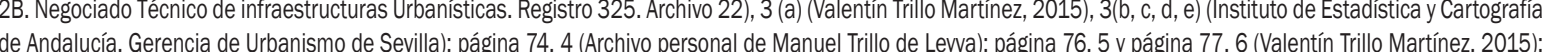
agina 78, 7 (a, b) (Archivo personal de Manuel Trillo de Leyva), 8 (Archivo personal de Manuel Trillo de Levva); pagina 78, 9 (a))(FDASS/COAS. Exxpediente 103802, caja 2454. Proyecto de Ejecución); página 80, 10 (Valentin Trillo Martinez, 2015); página 81, 11 (a) (Manu Trillo), 11 (b). Valentin Trillo Martínez, 2015); página 82, 12 y 83,

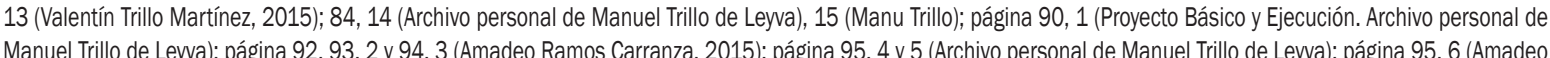

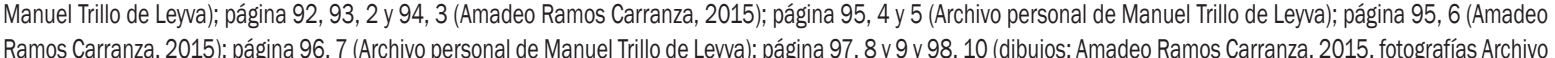
personal de Manuel ITillo de Levva); pagina 99, 11 (izda: Amadeo Ramos Carranza, 2015; dcha: A Archivo personal de Manuel Trillo de Levwa); página 100, 12 (Amadeo

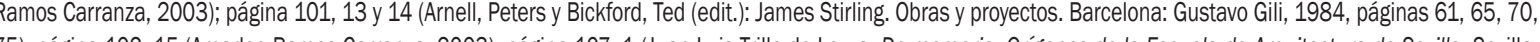
15); pägina 102, 15 (Amadeo Ramos Carranza, 2003); paginna 107, 1 Juan Luis Trillo de Leya: De memoria. Orígenes de la Escuela de Arquitectura de Sevilla. Sevilla:

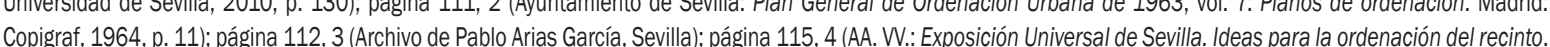

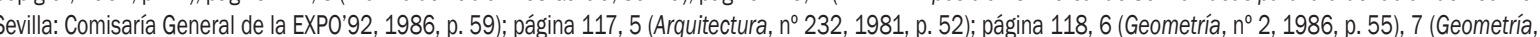

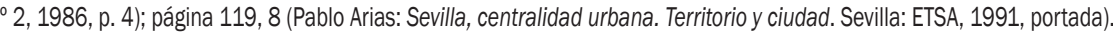

